\title{
Brivaracetam: a newly approved medication for epilepsy
}

\author{
Joel M Oster*,1,2 \\ ${ }^{1}$ Department of Neurology, Tufts Medical Center, Tufts University School of Medicine, Boston, MA 02111, USA \\ ${ }^{2}$ Lahey Clinic, Burlington, MA 02111, USA \\ *Author for correspondence: Tel.: +1 617636 5848; Fax: +1 617636 8199; joster@tuftsmedicalcenter.org
}

Brivaracetam (BRV) in both the USA and EU was developed as a novel molecule for the adjunctive treatment of partial-onset (focal) seizures in patients $\geq 16$ years of age and as of September 2017 was approved for use as monotherapy in the USA uniquely as an antiseizure medication that may be prescribed without a dose finding uptitration. This article reviews BRV's pharmacology, efficacy, safety and adverse event profiles, along with the relevant and noted regulatory hurdles in the USA and the EU. Available postmarketing data will also be summarized. Approximately 3000 patients were studied over about 9 years in the clinical trial program illustrating that BRV has efficacy at 50-200 mg/day with an acceptable adverse event profile.

First draft submitted: 3 December 2015; Accepted for publication: 5 September 2018; Published online: 8 October 2018

Keywords: antiepileptic medication $\bullet$ antiseizure medication $\bullet$ brivaracetam • clinical trials • efficacy and safety • epilepsy • intractable epilepsy • pharmacokinetics • seizure disorders

\section{Historical notes}

Brivaracetam (BRV) is a novel molecule that was discovered and developed by UCB Pharmaceuticals of Brussels, Belgium (www.ucb.com), a global biopharmaceutical company [1-15]. BRV was approved by the EMA and US FDA in January 2016 and February 2016, respectively, initially as an adjunctive agent for the treatment of partial-onset seizures (POS) in patients $\geq 16$ years of age and BRV launched in the USA in May 2016 for that indication [4-8]. Approximately 18 months later by September 2017, BRV was approved by the FDA as monotherapy treatment for POS that may uniquely be prescribed without a dose-finding uptitration [4-8].

This article overviews the historical development of BRV [1-15]. BRV's potential place in the current clinical market, which contains a persistent epidemiology of intractable epilepsy despite the existence of numerous products already available, will be discussed [16-22]. The article summarizes the major neurochemistry and pharmacology of BRV highlighting the relevant preclinical data [23-47]. This work identifies the significance of the clinical trial data and program and overviews the relevant issues surrounding regulatory affairs [43-49]. Safety and tolerability and a review of adverse events or treatment-emergent adverse events (AEs and TEAEs) of BRV are discussed [48-57]. Articles published peri- and post-launch of BRV are identified highlighting recent developments [58-83].

This article reviews MEDLINE-indexed journals cross-referenced with information available in the public domain regarding the 31 USA and 18 European clinical trials from the FDA and EMA [5,6]. The clinical trial program included approximately 3000 patients along with subsets including intractable patients, patients with and without secondarily generalized seizures, and patients that have had prior exposure to levetiracetam (LEV), a similar molecule already prescribed in the world marketplace $[1,2,4-8,48-56]$, see Tables $1-5$ and Discussion section.

The patients in the clinical trials to date have an approximate aggregate 9-year experience of BRV exposure [48,48-56]. Clinical trial experience has been complex and initially primary end points were not met in some of the Phase II and early Phase III trials likely due to analyses of less than $50 \mathrm{mg} /$ day dosing, which subsequently has been shown in that range to not have significant responder rates for treating POS in statistical analysis [48-56]. This article summarizes how the evolution and the compendium of all of the Phase III trials identify clinical efficacy for BRV in the 50-200 mg/day dose range and will outline the analysis of the AEs and TEAEs in the pivotal Phase IIb and Phase III trials to identify that those experienced in $\geq 5 \%$ of patients are generally mild and consist of somnolence,

Future s Medicine 
Table 1. Molecular summary for brivaracetam.

\begin{tabular}{l}
\hline Molecular structure \\
\hline Molar mass \\
\hline IUPAC name \\
\hline Discovery and development by: \\
\hline What is it
\end{tabular}

Summary of clinical trial development

Bioavailability/pharmacokinetics

Protein binding

Half-life

Metabolism and special populations
$\mathrm{C}_{11} \mathrm{H}_{2} \mathrm{O} \mathrm{N} \mathrm{O}_{2}$

$212.15 \mathrm{~g} / \mathrm{mol}$

(2S)-2-[(4R)-2-oxo-4-propylpyrrolidin-1-yl] butanamide

UCB Pharma

4- $n$-propyl analog of levetiracetam (KEPPRA/LEV) previously developed on worldwide pharmaceutical market. LEV and BRV are racetam derivatives used as antiepileptic medications. BRV trade nam: BRIVIACT.

US: Schedule V, pregnancy category $C$

- BRV's mechanism of action is thought be similar to that of LEV, which binds to the ubiquitous SV2A with multiple fold affinity and demonstrates faster brain bioavailability compared with LEV

- BRV is highly selective for SV2A and lacks activity at other receptor types such as AMPA, where LEV does have activity

- Literature review notes activity in myoclonus/Unverricht-Lundborg myoclonus

- BRV suppresses GABA inhibitors

- Protection from seizures in models of photomyoclonic response is noted

- BRV inhibits epileptiform discharges in various seizure models/neural preparations/animal models of status epilepticus

- Antiepileptic properties are noted in the electroshock model of seizures, audiogenic seizures and GAERS model

See Table 3 - there are currently noted 31 US FDA clinical trials and 18 European EUDRA clinical trials since discovery

Water soluble/nearly $100 \%$ with linear pharmacokinetics

$<20 \%$

6-11 h, geometric mean: $9 \mathrm{~h}$

- The major metabolism is hydrolysis of the BRV acetamide group to a carboxylic acid mediated by amide hydrolase. The secondary pathway is omega- 1 hydroxylation of the propyl side chain, which is mediated by CYP2C19

- Weight, use of other enzyme inducing agents/antiepileptic medications can cause mainly a mild reduction in blood levels

- Populations with impaired renal function may have effects not requiring dose adjustment, $>95 \%$ of BRV excreted by kidneys. Due to lack of data, BRV is not recommended in end-stage renal disease patients undergoing dialysis

- Hepatic impairment can significantly increase blood levels, caution is advised in dosing this group of patients, lower daily max dosing at $150 \mathrm{mg} /$ day should be considered. No dose adjustment needed in patients $\geq 65$ years of age although clinical experience in that group is limited

- Trials and data suggest minor effects on oral contraceptives and anticoagulants and low drug/drug interactions, although some special cases might exist. See text

Dosing/formulations

Based on clinical trial data, recommended starting dose is either 50 or $100 \mathrm{mg} /$ day. Therapeutic dose range is based on well-studied dosing from the clinical trial program and literature review ranging from 50 to $200 \mathrm{mg} /$ day. Tablets in strength 10, 25, 50, 75 and $100 \mathrm{mg}$ currently available and may be taken with or without food. iv. form available in clinical trials

Drug/drug interactions/precautions in special circumstances

Caveats: it is unknown whether BRV is excreted in

human breast milk, although noted in rats.

- Theoretical issue of increasing CBZ epoxide excreted in breast milk noted, insufficient evidence in human population

- No effects on fertility noted in humans, no effects in rats noted

- BRV thought to have minor or moderate influence on ability to operate motor vehicles and machines

- In USA - pregnancy category C - there is a limited amount of data from the use in pregnant women, no data on placental transfer but BRV shown to cross placenta in rats. Animal studies so far have not detected any teratogenic potential of BRV/risks unknown

- As a precaution, BRV should not be used during

pregnancy unless clinically necessary (benefit to mother clearly outweighs potential risks to fetus)

- Coadministration with phenytoin may increase phenytoin levels

- BRV provides no added benefit or safety or tolerability concerns noted with coadministration with LEV - Coadministration with CBZ may increase exposure to the CBZ-epoxide and could theoretically lead to reduced CBZ tolerability

- Coadministration with rifamycin may reduce BRV bioavailability by approximately $45 \%$

- BRV may theoretically inhibit CYP2C19 metabolism and increase medications metabolized by that mechanism - Theoretically, strong enzyme inducers (phenytoin, CBZ, phenobarbital) may reduce BRV bioavailability, extent is unknown, generally dose alteration seems not required

- In a study of BRV $200 \mathrm{mg}$ and ethanol $0.6 \mathrm{~g} / \mathrm{I}$ infusion, BRV may increase the negative effects of alcohol on psychomotor function, attention and memory, so intake with alcohol is not recommended

- Risk of CYP3A4 interactions considered low/study at 50 and $150 \mathrm{mg}$ BRV and midazolam showed no significant effects on bioavailability

- BRV may increase plasma concentrations of medicinal products involving OAT3

- BRV coadministration with oral contraceptives did not influence pharmacokinetics, even at $400 \mathrm{mg} / \mathrm{day}$, no impact on suppression of ovulation noted, no effects on estradiol, progesterone, luteinizing hormone, follicle stimulating hormone and sex hormone-binding globulin

- Otherwise overall clinical clinically significant drug/drug interactions have not been noted on other antiepileptic drugs from pooled data analysis of Phase II-III studies of the clinical trial program

BRV: Brivaracetam; CBZ: Carbamazepine; GABA: Gamma-aminobutyric acid; GAERS: Generalized absence epilepsy in rats from strasburg; iv.: Intravenous; IUPAC: International Union of Pure and Applied Chemistry; LEV: Levetiracetam. 
Table 2. Receptors and antiseizure medications.

\begin{tabular}{|c|c|}
\hline Receptor/mechanism & Edications \\
\hline Sodium channel blocker & $\begin{array}{l}\text { Phenytoin, carbamazepine, oxcarbazepine, eslicarbamazepine, lamotrigine, } \\
\text { topiramate }\end{array}$ \\
\hline Increase slow inactivation of sodium channel & Lacosamide, rufinamide \\
\hline GABA receptor agonist & Phenobarbital, benzodiazepines, clobazam \\
\hline NMDA receptor blockers & Felbatol \\
\hline AMPA receptor blocker & Perampanel, topiramate \\
\hline T-calcium channel blockers & Ethosuximide and valproate \\
\hline $\mathrm{H}$-current modulating effects & Gabapentin, lamotrigine \\
\hline $\mathrm{N}$ - and L-calcium channel blockers & Lamotrigine, valproic acid topiramate, zonisamide \\
\hline Carbonic anhydrase inhibitors & Zonisamide, topiramate \\
\hline KCNQ [Kv7] opener & Ezogabine/retigabine \\
\hline$\alpha 2 \delta$ protein & Gabapentin, pregabalin \\
\hline Collapsing response-mediating protein-2 & Lacosamide \\
\hline SV2A receptor & Levetiracetam and brivaracetam \\
\hline $\begin{array}{l}\text { GABA: Gamma-aminobutyric acid. } \\
\text { Reader is referred to other references for further information }[2,3,22] \text {. }\end{array}$ & \\
\hline
\end{tabular}

dizziness, fatigue, influenza like syndromes, insomnia, nasopharyngitis and other symptoms [4-8,48-56]. A discussion about treatment-emergent nonpsychotic behavioral events is also included and how this issue may be different than another medication LEV in the same class that has already been released to world markets [48-56].

A New Drug Application in the USA and a Marketing Authorization Application in the EU were submitted to those respective agencies and remained under consideration for approval until recently [4-8]. The Phase IIB and Phase III clinical trial dataset and investigations along with preliminary results began and became available by approximately 2007.

By January 2015, BRV progressed to the final review analyses by both the FDA and EMA for potential approval as an adjunctive treatment for focal (POS) in adult patients with epilepsy 16 years or older [4-8]. In November 2015, BRV received positive comments from the EMA and Committee for Human Medicinal Products (CHMP in the EU) and launched in the UK in January 2016. BRV was approved under the trade name 'Briviact' in February 2016 in the USA [4-8]. In September 2017, BRV was approved by the FDA for use in monotherapy for POS and may be initiated at a therapeutic dose without uptitration [4-8].

Presently, multiple ongoing studies as part of the clinical trials in the US and EU of BRV are still in effect, including multiple open-label studies to assess the long-term safety and efficacy of BRV [4-8]. The reader is referred to the Discussion section and Tables 3 and 4 that highlight the trials that form the previous and ongoing BRV research and development program.

\section{Significance \& place in the worldwide antiseizure medication market}

The first antiseizure medication was first noted around the late 1850s, with the discovery and development of bromide salts/triple bromide therapy [1-3,9]. After bromides became available, in the early part of the 1900s, phenobarbital and phenytoin and multiple new antiseizure/epileptic drugs (AEDs) were developed and introduced in the USA and worldwide markets over the next few decades. Although many medications may have been discovered by chance, others were developed utilizing synthetic organic chemistry or methodologies known as rational (or targeted) drug discovery [3]. In this endeavor, various manufactured compounds are tested and modified within a strategy program or paradigm of investigation, using predictive animal research or biochemical models [2]. Conversely, BRV ensued by studying compounds that optimized the pharmacodynamic activity atSV2A - the molecular target $[1,2,9-15]$.

BRV is related structurally and mechanistically to the action of LEV (brand name, Keppra) - ((S)- $\alpha$-ethyl-2oxopyrrolidine acetamide) [1-3,9-15]. BRV is a pyrrolidone that is structurally related to LEV and piracetam and the three compounds bind to the same molecular although with different affinities - BRV having the highest affinity of these [1-3,9-15]. BRV binds at an approximately $90 \mathrm{kDa}$ neuron-specific protein that is widely distributed in the brain and highly enriched in synaptic vesicles. The protein was identified as SV2A that is believed to be involved with neurotransmitter release. There is a high correlation between the binding affinities of a series of LEV analogs 
Table 3. Overview of the extensive clinical research trial program for the development of brivaracetam.

\begin{tabular}{|c|c|c|c|}
\hline NCT/US designation & Dates & Status & $\begin{array}{l}\text { Results published or significance EU/EUDRACT } \\
\text { designation if any }\end{array}$ \\
\hline $\begin{array}{l}\text { NСТ01796899: Comparison of brivaracetam oral } \\
\text { tablet }(10,50,75 \text { and } 100 \mathrm{mg} \text { ) and brivaracetam } \\
\text { injection }(100 \mathrm{mg}) \text { in healthy volunteers }\end{array}$ & $\begin{array}{l}\text { First received: } 19 \text { February } \\
2013 \\
\text { Last updated: } 15 \text { April } \\
2013\end{array}$ & Completed & $\begin{array}{l}\text { Phase I trial } \\
\text { (2006), Sunday, } 3 \text { December 2006; Poster Session II } \\
\text { 7:30 a.m.-4:30 p.m. [69] } \\
\text { Other study numbers EP0007, 2012-001358-25, } \\
\text { study recruited in The Netherlands }\end{array}$ \\
\hline $\begin{array}{l}\text { NCT00357669: brivaracetam as add-on treatment } \\
\text { of Unverricht-Lundborg disease in adolescents } \\
\text { and adults }\end{array}$ & $\begin{array}{l}\text { First received: } 25 \text { July } \\
\text { 2006, completed } 2007 \\
\text { Last updated: } 18 \text { May } 2015\end{array}$ & Completed & $\begin{array}{l}\text { Phase III trial } \\
\text { Finland, France, Italy, The Netherlands, Sweden, } \\
\text { Tunisia } \\
\text { N01187, 2006-000169-12 } \\
\text { [61] }\end{array}$ \\
\hline $\begin{array}{l}\text { NCT00490035: double-blind, randomized study } \\
\text { evaluating the efficacy and safety of brivaracetam } \\
\text { in adults with partial-onset seizures }\end{array}$ & $\begin{array}{l}\text { First received: } 21 \text { June } \\
2007 \\
\text { Last updated: } 17 \text { March } \\
2017\end{array}$ & Completed & $\begin{array}{l}\text { Phase III trial } \\
\text { Belgium, Finland, France, Germany, Hungary, } \\
\text { India, Italy, The Netherlands, Poland, Spain, } \\
\text { Switzerland, UK } \\
\text { N01252, 2006-006344-59 } \\
\text { [50] }\end{array}$ \\
\hline $\begin{array}{l}\text { NCT00175825: a dose-ranging study with } \\
\text { brivaracetam in patients from } 16 \text { to } 65 \text { years with } \\
\text { refractory partial-onset seizures }\end{array}$ & $\begin{array}{l}\text { First received: } 9 \\
\text { September } 2005 \\
\text { Last updated: } 29 \text { August } \\
2012\end{array}$ & Completed & $\begin{array}{l}\text { Corresponds with UCB Protocol N01193 } \\
\text { Phase II trial } \\
\text { USA, Brazil, India, Mexico } \\
\text { [48] }\end{array}$ \\
\hline $\begin{array}{l}\text { NCT02602860: a brain imaging study with } \\
\text { positron emission tomography and the } \\
\text { radiotracer [11C] UCB-J to estimate how fast } \\
\text { brivaracetam and levetiracetam enter the brain in } \\
\text { healthy volunteers }\end{array}$ & $\begin{array}{l}\text { First received: } 6 \text { November } \\
2015 \\
\text { Last updated: } 12 \text { May } 2017\end{array}$ & Currently recruiting & Phase I, US study EP0074 \\
\hline $\begin{array}{l}\text { NСT01710670: a study to investigate the } \\
\text { drug-drug interactions of brivaracetam and } \\
\text { ethanol in healthy male subjects }\end{array}$ & $\begin{array}{l}\text { First received: } 17 \text { October } \\
2012 \\
\text { Last updated: } 10 \\
\text { December } 2012\end{array}$ & Completed & Phase I, The Netherlands, EP0041, 2012-002591-14 \\
\hline $\begin{array}{l}\text { NCT00464269: double-blind, randomized study } \\
\text { evaluating the efficacy and safety of brivaracetam } \\
\text { in adults with partial-onset seizures }\end{array}$ & $\begin{array}{l}\text { First received: } 19 \text { April } \\
2007 \text { updated: } 17 \text { March } \\
2017\end{array}$ & Completed & $\begin{array}{l}\text { [51] } \\
\text { [59] } \\
\text { Phase III study } \\
400 \text { Subjects } \\
\text { USA, Australia, Brazil, Canada, Mexico } \\
\text { N01253, 2006-006345-14 }\end{array}$ \\
\hline $\begin{array}{l}\text { NСT01405508: safety and tolerability of } \\
\text { intravenous brivaracetam (infusion or bolus) as } \\
\text { adjunctive antiepileptic therapy }\end{array}$ & $\begin{array}{l}\text { First received: } 27 \text { July } 2011 \\
\text { Last updated: } 1 \text { August } \\
2016\end{array}$ & Completed & $\begin{array}{l}\text { Phase III } \\
\text { USA, Czech Republic, Germany, Poland } \\
\text { N01258, 2008-004714-27 } \\
{[46]} \\
{[69]}\end{array}$ \\
\hline $\begin{array}{l}\text { NCT01261325: brivaracetam efficacy and safety } \\
\text { study in subjects with partial-onset seizures } \\
\text { 'BRITE }^{\text {TM }} \text { study }\end{array}$ & $\begin{array}{l}\text { First received: } 9 \text { December } \\
2010 \\
\text { Last updated: } 23 \text { July } 2017\end{array}$ & Completed & $\begin{array}{l}\text { Phase III } \\
768 \text { Enrollment } \\
\text { USA, Austria, Belgium, Brazil, Bulgaria, Canada, } \\
\text { Czech Republic, Estonia, Finland, France, } \\
\text { Germany, Hong Kong, Hungary, India, Italy, Japan, } \\
\text { Korea, Republic of, Latvia, Lithuania, Mexico, The } \\
\text { Netherlands, Poland, Puerto Rico, Russian } \\
\text { Federation, Spain, Sweden, Taiwan, UK } \\
\text { N01358, 2010-019361-28 } \\
\text { Resulted in } \\
\text { [53] } \\
\text { [59] } \\
\text { [60] }\end{array}$ \\
\hline
\end{tabular}

$\dagger^{\dagger}$ Denotes studies that in particularly have been useful in estimating AEs, TEAEs, SAEs for the trial, see the Discussion setion in the text.

The table notes the current 31 US clinical trials performed and or registered in the US by online search with equivalent matching of the 18 EU clinical trials and cross-references where appropriate and available. Data for this table were compiled and organized from online review of www.clinicaltrials.gov and www.clinicaltrialsregister.eu of publically available online information regarding clinical trials for brivaracetam. Most recent access was on 10/8/2017 by the author [5,6]. Data obtained for the table were also obtained by Medline/PubMed search using NCT numbers and EU clinical trial numbers to track any publications/presentations/updates in the far right column for cross-referencing as available. Additional information for this table was also obtained searching UCB's website at www.ucb.com/rd/clinical-study-information/brivaracetam, which contains noted subset(s) of the clinical trials. In summary, the purpose of the above was done to obtain the current and most up-to-date available data regarding the types and scope of trials both completed and still enrolling in order to outline the extensive research program for brivaracetam at the time of this article's publication. These trials give an overview as to what types of emerging data may be already or imminently available from analysis and follow-up of this multifaceted clinical research program on the development of brivaracetam. See the latter portion of the table to identify the currently registered postmarketing studies.

AE: Adverse event; AED: Antiepileptic drug; BRV: Brivaracetam; EMU: Epilepsy monitoring unit; iv.: Intravenous; LEV: Levetiracetam; LZP: Lorazepam; NIS: Noninterventional study; PGS: Primary generalized seizure; PI: Package insert; POS: Partial-onset seizure; SAE: Serious adverse event; TEAE: Treatment-emergent adverse event. 
Table 3. Overview of the extensive clinical research trial program for the development of brivaracetam. (cont.).

NCT/US designation
NCT01339559: brivaracetam safety and efficacy
follow-up study in subjects with epilepsy part of
'BRITE'T' STUDY - Phase III open-label multicenter
follow-up study in evaluating long-term safety
and efficacy of BRV used as adjunctive treatment
in patients $\geq 16$ years of age
Flexible dosing up to $200 \mathrm{mg} /$ day $^{\dagger}$

NCT00761774: an open-label, multinational, multicenter, follow-up study to evaluate the long-term safety and efficacy of brivaracetam ${ }^{\dagger}$
Flexible dosing up to $200 \mathrm{mg}$ /day
Dates

First received: 19 April 2011

Last updated: 6 October 2017
Status

Active not recruiting designation if any

Phase III open-label

Estimated enrollment 820

USA, Austria, Belgium, Brazil, Bulgaria, Canada, Czech Republic, Estonia, Finland, France, Germany, Hong Kong, Hungary, India, Italy, Japan, Korea, Republic of, Latvia, Lithuania, Mexico, The Netherlands, Poland, Puerto Rico, Russian Federation, Spain, Sweden, Taiwan, UK Reviewing AEs, TEAEs, SAEs up to 4 years of study N01379, UCB34714, 2010-020345-27
First received: 26 September 2008 Last updated: 13 April 2017
Completed/not recruiting N01315 Protocol

Phase III study

USA, Australia, Belgium, Canada, Czech Republic, France, Germany, Hungary, Italy, Spain, Sweden 2008-001433-98

Evaluated AEs, TEAEs, SAEs, follow-up to 9 years of study

NCT00160667: a study assessing efficacy of brivaracetam in subjects with persistent pain after shingles (post-herpetic neuralgia) Last updated: 18 October

NCT00175929: a study of brivaracetam in subjects with partial-onset seizures brivaracetam and phenytoin in subjects with nonconvulsive electrographic seizures

NCT01728077: evaluation of long-term safety, and efficacy of brivaracetam (BRV) used as adjunctive treatment in subjects with epilepsy

An open-label multicenter follow-up study to evaluate the long-term safety and efficacy of BRV used as adjunctive treatment in subjects

aged $\geq 16$ years old with epilepsy, Phase $\mathrm{IIIb}^{\dagger}$

NCT01364597: open-label long-term study of adjunctive brivaracetam in pediatric subjects with epilepsy

NCT00504881: brivaracetam as add-on treatment in adolescents and adults suffering from epilepsy This study will compare the safety and efficacy of BRV at flexible dose with placebo in subjects suffering from epilepsy

\section{First received: 8}

September 2005 2010

First received: 9 September 2005 Last updated: 13 April 2015

First received: 20 February Terminated 2014

Last updated: 2 August 2016

\section{First received: 26 October} 2012 Last updated: 1 September 2017

Completed

Phase II study

N01162, EudraCT 2004-000975-32

Belgium, Bulgaria, Czech Republic, France, Germany, Poland, Serbia, Slovakia, Spain, UK

\section{Protocol N0114}

Phase II study

N01114, EudraCT2004-001856-35 [49]

Terminated due to low enrollment, no safety issues

Phase II

US study

N01394

Completed/not recruiting Phase IIIb

Estimated enrollment 650

USA, France, Germany, Spain

N01372, UCB34714, 2012-000827-42

\section{First received: 31 May 2011 Enrolling by invitation Last updated: 11 September 2017 \\ only Estimated completion date $10 / 2021$}

\section{Phase III}

Estimated enrollment 600

USA, Belgium, Czech Republic, Mexico, Poland,

Spain

N01266, 2011-000374-60

\section{First received: 19 July 2007 Completed}

Last updated: 17 March

Phase III Trial

Enrollment 480

N01254 protocol: [52]

Austria, Belgium, Czech Republic, Germany, Hong

Kong, India, Italy, Korea, Republic of, Norway,

Russian Federation, Singapore, South Africa,

Sweden, Taiwan, Ukraine

2006-006346-34

† Denotes studies that in particularly have been useful in estimating AEs, TEAEs, SAEs for the trial, see the Discussion setion in the text.

The table notes the current 31 US clinical trials performed and or registered in the US by online search with equivalent matching of the 18 EU clinical trials and cross-references where appropriate and available. Data for this table were compiled and organized from online review of www.clinicaltrials.gov and www.clinicaltrialsregister.eu of publically available online information regarding clinical trials for brivaracetam. Most recent access was on 10/8/2017 by the author [5,6]. Data obtained for the table were also obtained by Medline/PubMed search using NCT numbers and EU clinical trial numbers to track any publications/presentations/updates in the far right column for cross-referencing as available. Additional information for this table was also obtained searching UCB's website at www.ucb.com/rd/clinical-study-information/brivaracetam, which contains noted subset(s) of the clinical trials. In summary, the purpose of the above was done to obtain the current and most up-to-date available data regarding the types and scope of trials both completed and still enrolling in order to outline the extensive research program for brivaracetam at the time of this article's publication. These trials give an overview as to what types of emerging data may be already or imminently available from analysis and follow-up of this multifaceted clinical research program on the development of brivaracetam. See the latter portion of the table to identify the currently registered postmarketing studies.

AE: Adverse event; AED: Antiepileptic drug; BRV: Brivaracetam; EMU: Epilepsy monitoring unit; iv.: Intravenous; LEV: Levetiracetam; LZP: Lorazepam; NIS: Noninterventional study; PGS: Primary generalized seizure; PI: Package insert; POS: Partial-onset seizure; SAE: Serious adverse event; TEAE: Treatment-emergent adverse event. 
Table 3. Overview of the extensive clinical research trial program for the development of brivaracetam. (cont.)

\begin{tabular}{|c|c|c|c|}
\hline NCT/US designation & Dates & Status & $\begin{array}{l}\text { Results published or significance EU/EUDRACT } \\
\text { designation if any }\end{array}$ \\
\hline $\begin{array}{l}\text { NCT00150800: open-label follow-up trial to } \\
\text { evaluate long-term safety and efficacy of } \\
\text { brivaracetam in subjects aged } 16 \text { years or older } \\
\text { suffering from epilepsy }{ }^{\dagger}\end{array}$ & $\begin{array}{l}\text { First received: } 6 \\
\text { September } 2005 \\
\text { Last updated: } 2 \text { October } \\
2017\end{array}$ & Completed/not recruiting & $\begin{array}{l}\text { Phase III } \\
\text { Enrollment } 668 \text { patients, September } 2017 \text { is the } \\
\text { final data collection date for primary outcome } \\
\text { measure } \\
\text { Estimated completion } 2017 \\
\text { USA, Australia, Brazil, Canada, India, Mexico } \\
\text { N01199, N01193, N01252, N01253, N01254 }\end{array}$ \\
\hline $\begin{array}{l}\text { NCT00736931: clinical study in healthy volunteers } \\
\text { to investigate the neurocognitive effects of a new } \\
\text { antiepileptic drug: brivaracetam }\end{array}$ & $\begin{array}{l}\text { First received: } 14 \text { August } \\
2008 \\
\text { Last updated: } 28 \text { May } 2012\end{array}$ & Completed & $\begin{array}{l}\text { Phase I trial } \\
20 \text { Patients } \\
\text { [57] } \\
\text { US study only } \\
\text { N01297 }\end{array}$ \\
\hline $\begin{array}{l}\text { NCT00698581: a double-blind, randomized } \\
\text { conversion to monotherapy study to evaluate the } \\
\text { efficacy and safety of brivaracetam in subjects } \\
\text { ( } \geq 16-75 \text { years old) with partial-onset seizures }\end{array}$ & $\begin{array}{l}\text { First received: } 12 \text { June } \\
2008 \\
\text { Last updated: } 13 \text { March } \\
2017\end{array}$ & $\begin{array}{l}\text { Terminated } \\
\text { An interim analysis } \\
\text { revealed the study was } \\
\text { unlikely to attain a } \\
\text { positive outcome for the } \\
\text { primary efficacy analysis. } \\
\text { No safety concerns were } \\
\text { detected }\end{array}$ & $\begin{array}{l}\text { [54] } \\
\text { This article reviews this and similar study designs } \\
\text { Phase III study } \\
\text { USA, Australia, Belgium, Canada, Czech Republic, } \\
\text { Germany, Sweden } \\
\text { N01276, RPCE07F1214, 2008-000144-14 }\end{array}$ \\
\hline $\begin{array}{l}\text { NCT00422422: open-label, pharmacokinetic, } \\
\text { safety and efficacy study of adjunctive } \\
\text { brivaracetam in children with epilepsy }\end{array}$ & $\begin{array}{l}\text { First received: } 10 \text { January } \\
2007 \\
\text { Last updated: } 9 \text { January } \\
2017\end{array}$ & Completed & $\begin{array}{l}\text { Phase II trial } \\
\text { USA, Belgium, Czech Republic, Mexico, Poland, } \\
\text { Spain } \\
\text { N01263, 2006-006536-22 } \\
\text { [69] }\end{array}$ \\
\hline $\begin{array}{l}\text { NCT00175916: follow-up trial to evaluate } \\
\text { long-term safety and efficacy of brivaracetam in } \\
\text { subjects suffering from epilepsy } \\
\text { This trial, evaluating the long-term safety and } \\
\text { tolerability of BRV, will give subjects suffering } \\
\text { from epilepsy, who may have benefited from BRV, } \\
\text { the opportunity to continue the treatment. The } \\
\text { study will also evaluate the maintenance of } \\
\text { efficacy over time of BRV for subjects with } \\
\text { POS/PGS }\end{array}$ & $\begin{array}{l}\text { First received: } 9 \\
\text { September } 2005 \\
\text { Last updated: } 1 \text { September } \\
2017\end{array}$ & Active not recruiting & $\begin{array}{l}\text { Phase III } \\
853 \text { Patients } \\
\text { N01125, 2004-002140-10 } \\
\text { USA, Austria, Belgium, Canada, Czech Republic, } \\
\text { Finland, France, Germany, Hong Kong, Hungary, } \\
\text { Israel, Italy, Korea, Republic of, The Netherlands, } \\
\text { Norway, Poland, Russian Federation, Serbia, } \\
\text { Singapore, South Africa, Spain, Sweden, } \\
\text { Switzerland, Taiwan, Tunisia, Ukraine }\end{array}$ \\
\hline $\begin{array}{l}\text { NCT00368251: brivaracetam as add-on treatment } \\
\text { of Unverricht-Lundborg disease (ULD) in } \\
\text { adolescents and adults }\end{array}$ & $\begin{array}{l}\text { First received: } 23 \text { August } \\
2006 \\
\text { Last updated: } 1 \text { August } \\
2017\end{array}$ & Completed & $\begin{array}{l}\text { Phase III trial } \\
\text { N01236, RPCE06C2320, 2006-001536-46 } \\
\text { USA, Canada, Finland, France, Israel, Russian } \\
\text { Federation, Serbia, Tunisia } \\
{[61,85,86]}\end{array}$ \\
\hline $\begin{array}{l}\text { NCT00401648: effect of brivaracetam in } \\
\text { photosensitive epileptic subjects }\end{array}$ & $\begin{array}{l}\text { First received: } 17 \\
\text { November } 2006 \\
\text { Last updated: } 30 \\
\text { November } 2009\end{array}$ & Completed & $\begin{array}{l}\text { Phase II Trial } \\
\text { N01069, Protocol Number RPCE02D2304 } \\
\text { France, Germany [39] }\end{array}$ \\
\hline $\begin{array}{l}\text { NCT00699283: a double-blind, randomized } \\
\text { conversion to monotherapy study to evaluate the } \\
\text { efficacy and safety of brivaracetam in subjects } \\
\text { ( } \geq 16-75 \text { years old) with partial-onset seizures }\end{array}$ & $\begin{array}{l}\text { First received: } 12 \text { June } \\
2008 \\
\text { Last updated: } 18 \text { January } \\
2017\end{array}$ & $\begin{array}{l}\text { This study has been } \\
\text { terminated } \\
\text { (An interim analysis } \\
\text { revealed the study was } \\
\text { unlikely to attain a } \\
\text { positive outcome for the } \\
\text { primary efficacy analysis. } \\
\text { No safety concerns were } \\
\text { detected) }\end{array}$ & $\begin{array}{l}\text { Review on this type of study design: [54] } \\
\text { Phase III trial } \\
\text { N01306, RPCE07F1216, 2008-000145-58 } \\
\text { USA, France, Germany, Hungary, Italy, Spain }\end{array}$ \\
\hline
\end{tabular}

$\dagger$ Denotes studies that in particularly have been useful in estimating AEs, TEAEs, SAEs for the trial, see the Discussion setion in the text.

The table notes the current 31 US clinical trials performed and or registered in the US by online search with equivalent matching of the 18 EU clinical trials and cross-references where appropriate and available. Data for this table were compiled and organized from online review of www.clinicaltrials.gov and www.clinicaltrialsregister.eu of publically available online information regarding clinical trials for brivaracetam. Most recent access was on 10/8/2017 by the author [5,6]. Data obtained for the table were also obtained by Medline/PubMed search using NCT numbers and EU clinical trial numbers to track any publications/presentations/updates in the far right column for cross-referencing as available. Additional information for this table was also obtained searching UCB's website at www.ucb.com/rd/clinical-study-information/brivaracetam, which contains noted subset(s) of the clinical trials. In summary, the purpose of the above was done to obtain the current and most up-to-date available data regarding the types and scope of trials both completed and still enrolling in order to outline the extensive research program for brivaracetam at the time of this article's publication. These trials give an overview as to what types of emerging data may be already or imminently available from analysis and follow-up of this multifaceted clinical research program on the development of brivaracetam. See the latter portion of the table to identify the currently registered postmarketing studies.

AE: Adverse event; AED: Antiepileptic drug; BRV: Brivaracetam; EMU: Epilepsy monitoring unit; iv.: Intravenous; LEV: Levetiracetam; LZP: Lorazepam; NIS: Noninterventional study; PGS: Primary generalized seizure; PI: Package insert; POS: Partial-onset seizure; SAE: Serious adverse event; TEAE: Treatment-emergent adverse event. 
Table 3. Overview of the extensive clinical research trial program for the development of brivaracetam. (cont.).

NCT/US designation

NCT01653262: effect of brivaracetam (BRV) on nonpsychotic behavioral side effects in subjects treated previously with levetiracetam (LEV): An open-label, multicenter, single-arm study to evaluate the reduction in nonpsychotic behavioral side effects in subjects with epilepsy switching from levetiracetam to brivaracetam due to nonpsychotic behavioral side effects Phase IIIb Brief summary

Trial N01395 is to evaluate the reduction of nonpsychotic behavioral side effects in subjects with epilepsy who switched to BRV $200 \mathrm{mg} /$ day after discontinuing LEV due to such side effects, as well as the efficacy, safety and tolerability of BRV. No statistical hypothesis testing will be performed NCT00426673: an open-label interaction study to look at the effects of brivaracetam on phenytoin when taken together in 15 adult patients with epilepsy

Dates Status

First received: 26 July 2012 Completed

Last updated: 15 August

2016

EudraCT Number: 2015-002756-27

Full title: a multicenter, open-label, single-arm study to evaluate the pharmacokinetics, efficacy, and safety of brivaracetam in neonates with repeated electroencephalographic seizures

\begin{tabular}{|c|c|c|}
\hline First received: 23 January & Completed & Phase I \\
\hline 2007 & & US study \\
\hline $\begin{array}{l}\text { Last updated: } 6 \text { March } \\
2008\end{array}$ & & N01172 protocol \\
\hline
\end{tabular}

Sponsor protocol number: Information not available N01349 Start date:

Information not available

in EudraCT
Results published or significance EU/EUDRACT designation if any

N01395: [19]

Phase III

2011-005177-23

\section{Postmarketing studies}

\section{NCT03250377}

An open-label, multicenter, follow-up study to evaluate the long-term safety and efficacy of brivaracetam used as adjunctive treatment in Japanese subjects $\geq 16$ years of age with partial seizures with or without secondary generalization ${ }^{\dagger}$

\author{
Submitted 7/31/2017 \\ Updated 9/15/2017 \\ Trial Started 8/5/2017 \\ Estimated primary \\ completion date \\ $11 / 30 / 2022$
}

\section{Posted 13 January 2017}

\begin{abstract}
Updated 9/15/2017
\end{abstract}
Active enrolling

Up to 60 patients ages

18-70 with concomitant

EMU admission
Medical condition: electroencephalographic neonatal seizures

Disease:

Population age: preterm newborn infants, newborns, infants and toddlers, under 18 Gender: male, female

Trial protocol: IE DE (ongoing) BE (ongoing) CZ (ongoing) $\mathrm{HU}$ (ongoing) $\mathrm{NL}$ (ongoing) FR Trial results: (no results available)
The purpose of the study is to evaluate the long-term safety and tolerability of BRV in focal epilepsy subjects with partial seizures and to evaluate the maintenance of efficacy of BRV over time

\section{NCT03021018}

The purpose of this study is to assess the efficacy of intravenous BRV compared with intravenous

LZP in subjects with epilepsy undergoing EMU evaluation who experience seizures that require prompt treatment

\section{NCT03083665}

A study to evaluate the efficacy and safety of adjunctive brivaracetam in Asian subjects ( $\geq 16-80$ years of age) with epilepsy

\section{Posted: 20 March 2017 Updated 15 September 2017}

Active enrolling Patients $18-80$ years of age, Phase III trial, estimated enrollment 504 patients, estimated completion date January 2020

\section{iv. formulation study}

Currently the author is participating at Tufts Medical Center in this clinical trial as PI at time of publication Phase II study

A randomized, double-blind, placebo-controlled multicenter, parallel-group study to evaluate the efficacy and safety of adjunctive BRV in Asian subjects ( $\geq 16-80$ years of age) with partial seizures with or without secondary generalization study also reviews secondary generalization despite current treatment with one or two concomitant AEDs and to assess the safety and tolerability of BRV in subjects $\geq 16-80$ years of age

†Denotes studies that in particularly have been useful in estimating AEs, TEAEs, SAEs for the trial, see the Discussion setion in the text.

The table notes the current 31 US clinical trials performed and or registered in the US by online search with equivalent matching of the 18 EU clinical trials and cross-references where appropriate and available. Data for this table were compiled and organized from online review of www.clinicaltrials.gov and www.clinicaltrialsregister.eu of publically available online information regarding clinical trials for brivaracetam. Most recent access was on 10/8/2017 by the author [5,6]. Data obtained for the table were also obtained by Medline/PubMed search using NCT numbers and EU clinical trial numbers to track any publications/presentations/updates in the far right column for cross-referencing as available. Additional information for this table was also obtained searching UCB's website at www.ucb.com/rd/clinical-study-information/brivaracetam, which contains noted subset(s) of the clinical trials. In summary, the purpose of the above was done to obtain the current and most up-to-date available data regarding the types and scope of trials both completed and still enrolling in order to outline the extensive research program for brivaracetam at the time of this article's publication. These trials give an overview as to what types of emerging data may be already or imminently available from analysis and follow-up of this multifaceted clinical research program on the development of brivaracetam. See the latter portion of the table to identify the currently registered postmarketing studies.

AE: Adverse event; AED: Antiepileptic drug; BRV: Brivaracetam; EMU: Epilepsy monitoring unit; iv.: Intravenous; LEV: Levetiracetam; LZP: Lorazepam; NIS: Noninterventional study; PGS: Primary generalized seizure; PI: Package insert; POS: Partial-onset seizure; SAE: Serious adverse event; TEAE: Treatment-emergent adverse event. 
Table 3. Overview of the extensive clinical research trial program for the development of brivaracetam. (cont.).

NCT/US designation
NCT02687711
add-on treatment for patients with epilepsy with
partial-onset seizures
A 12 -month noninterventional, postmarketing,
multicenter study to evaluate the effectiveness of
Briviact ${ }^{\circledR}$ (brivaracetam) as adjunctive therapy in
patients with epilepsy with partial-onset seizures
in daily clinical practice
Study also evaluates epilepsy with POS with or
without secondary generalization

Dates

First posted: 22 February 2016

Last update Posted: 15 September 2017
Status

Active enrolling

The primary objective of

this study is to determine

BRV retention over a

12-month period as a

measure of effectiveness

in a real-world setting.

The secondary objective of

this study is to assess

seizure control with BRV

treatment

Patients (male or female

$\geq 16$ years of age) with a

clinical diagnosis of

epilepsy with POS with or

without secondary

generalization. The

patients have never been

treated with BRV and the

decision by the treating

physician (neurologists) to

prescribe BRV is made

independently of the

participation in the study

and prior to enrollment

Patient meets the criteria

for treatment with BRV as

adjunctive therapy

according to the current in

Europe. Patient is using a

seizure diary as part of

their standard of care
Results published or significance EU/EUDRACT designation if any

Brief summary

Study is the first study after commercialization of

BRV. It is designed to collect real-world

information on the effectiveness of BRV in patients with POS epilepsy who are treated in standard clinical practice

EP0077 is a 12 months, multicenter, NIS conducted at specialized sites in approximately ten European countries. Patients will be treated according to usual medical diagnostic procedures and therapy; commercially available BRV will be prescribed according to normal clinical practice and the current summary of product characteristics in Europe for BRV. The prescription of BRV is clearly separated from the decision to include the patient in the study. No additional diagnostic or monitoring procedures are applied to the patients

$\dagger$ Denotes studies that in particularly have been useful in estimating AEs, TEAEs, SAEs for the trial, see the Discussion setion in the text.

The table notes the current 31 US clinical trials performed and or registered in the US by online search with equivalent matching of the 18 EU clinical trials and cross-references where appropriate and available. Data for this table were compiled and organized from online review of www.clinicaltrials.gov and www.clinicaltrialsregister.eu of publically available online information regarding clinical trials for brivaracetam. Most recent access was on 10/8/2017 by the author [5,6]. Data obtained for the table were also obtained by Medline/PubMed search using NCT numbers and EU clinical trial numbers to track any publications/presentations/updates in the far right column for cross-referencing as available. Additional information for this table was also obtained searching UCB's website at www.ucb.com/rd/clinical-study-information/brivaracetam, which contains noted subset(s) of the clinical trials. In summary, the purpose of the above was done to obtain the current and most up-to-date available data regarding the types and scope of trials both completed and still enrolling in order to outline the extensive research program for brivaracetam at the time of this article's publication. These trials give an overview as to what types of emerging data may be already or imminently available from analysis and follow-up of this multifaceted clinical research program on the development of brivaracetam. See the latter portion of the table to identify the currently registered postmarketing studies.

AE: Adverse event; AED: Antiepileptic drug; BRV: Brivaracetam; EMU: Epilepsy monitoring unit; iv.: Intravenous; LEV: Levetiracetam; LZP: Lorazepam; NIS: Noninterventional study; PGS: Primary generalized seizure; PI: Package insert; POS: Partial-onset seizure; SAE: Serious adverse event; TEAE: Treatment-emergent adverse event.

and their potencies for protection against audiogenic seizures, confirming that SV2A is the molecular target for anticonvulsant activity [1-3,9-12]. While SV2A appears to be involved in synaptic transmission by preparing vesicles for fusion, its functional role in synaptic transmission is not completely understood at present [1-3,9-12]. BRV binds to the SV2A protein with approximately ten- to 30 -fold higher than LEV and in animals - achieves bioavailability in the brain faster than LEV due to a higher CNS/brain permeability as compared with LEV in rodents [13-15]. LEV and BRV might be localized in the luminal side of recycling synaptic vesicles during endocytosis [58]. Although the above is known mechanistically about BRV, a review indicates that many of the molecular and pharmacologic mechanisms of previously approved medications may not have been known until many years after the launch and marketing of the product and that the exact mechanism of action may not actually be required by regulatory authorities [3-15].

Possibly due to the enhanced binding to SV2A, BRV seems to have additional effectiveness in suppressing epileptiform activity in various animal models, in particularly the rodent model, which has been most studied, when compared with LEV [11-13,22,23,25,28-31]. In general, LEV and BRV may reduce excitatory neurotransmitter release and enhance synaptic depression during seizures $[1-3,11,15,53]$. Although clinical trial data to date may not be totally clear on the head-to-head comparison of BRV versus LEV, it is speculated that BRV might have additional clinical therapeutic efficacy, and potentially a different, broader or enhanced clinical spectrum of action compared with LEV $[1-3,14,15,26]$. 


\section{Table 4. Clinical trial highlights - for detailed list of all clinical trials.}

Total of 31 US and 18 EU registered clinical trials, approximately 3000 patients total, some for approximately 9 years

A significant Phase II and a significant Phase III had issues of not meeting their primary end points but did so for secondary end points causing administrative hurdles at US FDA and EU, which assisted in development of later clinical trials. See below and the text

Two conversion to monotherapy trials were halted but not due to safety risks/see the text [4-8]

Trials on Unverricht-Lundborg myoclonus are at present not showing definite statistically significant clinical benefit in treatment of myoclonus and myoclonic epilepsy using the established rating scales $[4-8,61]$

There are two significant Phase Ilb studies of particular importance in the research and development of LEV

- Study as published by Van Paesschen et al. - failed to reach statistical significance for primary but not secondary end points [49]

- Study as published by French et al. - did reach statistical significance for primary and secondary end points [48]

There are currently four significant Phase III studies from the published literature

- UCB N0152 - same issues as above to the Van Paesschen et al.'s study - published by Ryvlin et al., involving 399 patients, failed to reach statistical significance for primary but not secondary end points [48]

- UCB N0153 - as published by Biton et al., 400 patients - did reach significance for primary and secondary end points [51]

- UCB N01254 - as published by Kwan et al., 480 patients - also did reach significance for primary and secondary end points [52]

$* * * *$ POOLED analysis of the above three Phase III studies indicate from review of data of published studies and on UCB websites that there is statistically significant efficacy (median \% reduction) compared with placebo [4-8,48-53]

- UCB N01358 - the most recent and largest Phase III Clinical protocol so far - still has data emerging, some reported by Klein et al., indicating favorable efficacy for primary and secondary end points as well as favorable safety outcomes in 768 patients, see the text [53], 88\% of patients entered long-term follow-up phase suggesting that treatment emergent adverse events were relatively low, see above for discussion

Further details about UCB Protocol N01252 - resulting in US NCT 004900035: this was a protocol denoting an evaluation of the efficacy and safety/tolerability of adjunctive BRV at 20,50 and $100 \mathrm{mg}$ /day compared with placebo over 12 weeks, in 399 randomized patients ( $\geq 16-70$ years) with partial-onset seizures not fully controlled despite treatment with 1-2 concomitant AEDs. This study as note by Ryvlin et al. did not meet its primary end point as noted above (\% reduction over placebo in baseline-adjusted focal seizure frequency per week) and was a significant hurdle in 2009 [50]

Further details about UCB Protocol N01253 - corresponding with US FDA 00464269 and EU 2006-006345-14: this experience was ultimately published by Biton et al. [51]. The purpose of this study was evaluation of the efficacy and safety/tolerability of adjunctive BRV at doses of 5, 20 and $50 \mathrm{mg} /$ day compared with placebo over 12 weeks, in 400 randomized patients ( $\geq 16-70$ years) with partial-onset seizures not fully controlled despite treatment with $1-2$ concomitant AEDs. This study achieved a statistically significant percentage reduction over placebo in baseline adjusted focal seizure frequency per week during the 12 -week treatment period at $50 \mathrm{mg} /$ day. The other doses of less than $50 \mathrm{mg} /$ day did not achieve significance, and the analysis and statistics analyzed and reflected the $\geq 50 \%$ responder rate. 392 patients comprised the intention to treat population, and 361 (91.2\%) of patients completed the study and most patients (78.3\%) received two concomitant AEDs and there was good tolerability, the reader is referred to the full reference [46].

Further details about UCB Protocol N01254 - corresponding with US FDA NCT 00504881 and EU 2006-006346-34: as published by Kwan et al., this was an evaluation of the safety and tolerability of adjunctive BRV given at individualized tailored doses between 20 and $150 \mathrm{mg} /$ day, compared with placebo over 16 weeks, in 480 randomized patients ( $\geq 16-70$ years) with uncontrolled epilepsy (up to $20 \%$ could be patients with generalized epilepsy), not fully controlled despite treatment with $1-3$ concomitant AEDs [52]. This study furthered supported the claims that BRV was effective and seemed to have efficacy reaching statistical significance treating focal and generalized seizures with favorable side effects [51,52]. Patients were randomized after a 4-week baseline (3:1) to BID BRV or PBO, initiated at 20 mg/day and increased as needed to $150 \mathrm{mg} /$ day during an 8-week dose-finding period followed by an 8-week maintenance period [52].

AED: Antiepileptic drug; BID: Twice daily; BRV: Brivaracetam; LEV: Levetiracetam; PBO: Placebo.

Despite numerous medications and treatments available, the prevalence of epilepsy is approximately 65 million worldwide, with about two million patients in the USA. [16-18]. The incidence of seizures is estimated at one in 26 (the highest in children and in older adults) according to the 2012 report from the Institute of Medicine [18]. As noted in the literature, LEV treatment became associated with nonpsychotic behavioral events, so motivation for finding a molecule, selective for the novel SV2A mechanism with fewer behavioral events seemed an impetus in BRV's development $[4-8,19]$. A recent open-label study in a small cohort of patients published in the premarketing phase by Yates et al. (which has been reconfirmed in the postmarketing phase/see below for review) indicated that a group of patients might benefit by switching to BRV if they had behavioral side effects on LEV [19].

There is vast literature on the definition and syndromes of epilepsy including what medications might be used and what agents might be used for rational polytherapy and the fact that seizures persist despite medications available along with surgical therapies $[14,20-47]$. As the clinical trials on surgery emerged in the literature over the last approximately 15 years, a fairly extensive clinical evaluation of BRV also emerged in the literature [23-47]. It was, therefore, speculated that BRV might reduce seizures in a vast number of such refractory epilepsy patients worldwide, or be useful to those with limiting side effects, since such patients were included within the selection of the BRV clinical trial program discussed below [1-15,48-54], and published and pooled data are becoming available identifying the fact that BRV has efficacy in refractory POS at least in a single Phase IIb $[43,59,61,62]$. The impact of $\mathrm{BRV}$ is thought to be therefore potentially significant when considering the epidemiology of both the prevalence and incidence of chronic epilepsy and persistence of refractory seizures. Although there are about 30 such medications available presently for treatment of seizure disorders at this point in time, there are still patients with uncontrolled seizures and side effects, and with the approval and launch of BRV, the full postmarketing significance and clinical 
Table 5. Adverse events from selected Phase II and III clinical trial data for brivaracetam.

\section{Safety issue \\ Summary by the numbers in clinical trial program so far: \\ 2388 subjects have received $B R V$, of whom \\ -1740 have been treated for $\geq 6$ months \\ -1363 for $\geq 12$ months \\ - 923 for $\geq 24$ months and 569 for \\ $\geq 60$ months ( 5 years) \\ Regarding open-label extension studies: generally BRV patients who were \\ followed-up in the open-label extension \\ studies for up to 8 years, the safety profile \\ was similar to that observed in the \\ short-term, placebo-controlled studies}

No statistical meaningful changes for these parameters throughout the clinical trial program for BRV at time of review

Neutropenia

Behavioral issues:

BRV package inserts have similar cautions to other medications used as AEDs; suicidal behavior and ideation: antiepileptic drugs, including BRV increase the risk of suicidal behavior and ideation. Monitoring patients who are taking BRV for the emergence or worsening of depression; unusual changes in mood or behavior; or suicidal thoughts, behavior or self-harm is/are advised. General advisement is to advise patients, their caregivers and/or families to be alert for these behavioral changes and report them immediately to a healthcare provider while taking BRV

Immediate (type I) hypersensitivity reactions: General recommendation: discontinue BRV if a patient develops a hypersensitivity reaction after treatment. BRV is contraindicated in patients with a prior hypersensitivity reaction to BRV itself or any of the inactive ingredients

\section{Special populations:}

The safety profile in children appears to be consistent with that known in adults. No data are available on neurodevelopment Currently, no clinical data are available in neonates

Safety in elderly appears similar to that of younger adult patients

General precautions advised

Overdose

\section{Detail}

The most frequently reported adverse reactions $(>10 \%)$ with BRV treatment were:

- Somnolence $(14.3 \%)$ and dizziness $(11.0 \%)$

They were usually mild to moderate in intensity

- Somnolence and fatigue $(8.2 \%)$ were reported at a higher incidence with increasing dose

- The types of adverse reactions reported during the first 7 days of treatment were similar to those reported for the overall treatment period

- BRV discontinuation rate due to adverse reactions was 3.5,3.4 and $4.0 \%$ for patients randomized to BRV at, respectively, the dose of 50,100 and $200 \mathrm{mg}$ /day and $1.7 \%$ for patients randomized to placebo in the general clinical trial program overall.

- The adverse reactions most frequently resulting in discontinuation of BRV therapy were dizziness $(0.8 \%)$ and convulsion $(0.8 \%)$

- Somnolence and fatigue-related adverse reactions were reported in $25 \%$ of patients taking at least $50 \mathrm{mg}$ per day of BRV compared with $14 \%$ of patients taking placebo. Dizziness and disturbance in gait and coordination were reported in $16 \%$ of patients taking at least $50 \mathrm{mg}$ per day of BRV compared with $10 \%$ of patients taking placebo

Blood chemistries, urinalysis, vital signs, EKG, physical examination, neurological examinations

Reported in $0.5 \%(6 / 1099)$ BRV patients and $0 \%(0 / 459)$ placebo patients. Four of these subjects had decreased neutrophil counts at baseline, and experienced additional decrease in neutrophil counts after initiation of BRV treatment. None of the six cases of neutropenia were severe, required any specific treatment or led to discontinuation of BRV and none had associated infections

Suicidal ideation has been reported in 0.3\% (3/1099) BRV patients and $0.7 \%$ (3/459) placebo patients. In the short-term clinical studies for BRV in epilepsy patients, there were no completed suicide and suicide attempt(s), however, both have been reported in open-label extension studies and general recommendations therefore are noted. Regarding nonpsychotic and psychotic symptoms, these events were reported in approximately $13 \%$ of patients taking at least $50 \mathrm{mg} /$ day of BRV compared with $8 \%$ of patients taking placebo. A total of $1.7 \%$ of adult patients taking BRV discontinued treatment due to psychiatric reactions compared with $1.3 \%$ of patients taking placebo. Patients and caregivers therefore advised patients to report these symptoms immediately to a healthcare provider

Reported patients (9/3022) BRV-treated patients during clinical development $(0.3 \%)$

Bronchospasm and angioedema have been reported
Pediatric program:

- There are limited safety data from open-label studies in children from 1 month to $<16$ years of age. A total of 152 children ( 1 month to $<16$ years of age) were treated with BRV in a pharmacokinetic study and the related follow-up study. From the limited available data, the most frequently reported TEAEs considered drug-related by the investigator were somnolence $(10 \%)$, decreased appetite $(8 \%)$, fatigue $(5 \%)$ and weight decreased $(5 \%)$ Elderly:

- Of the 130 elderly subjects enrolled in the BRV Phase II/III development program (44 with epilepsy), 100 were 65-74 years of age and 30 were $75-84$ years of age

UCB recommends to monitor patients for $A E s$ and advise patients not to drive or operate machinery until they have gained sufficient experience on BRV In general BRV should be withdrawn gradually because of the risk of increased seizure frequency and status epilepticus

There is limited clinical experience with BRV overdose in humans. Somnolence and dizziness have been reported in a healthy subject taking a single dose of $1400 \mathrm{mg} B R V$ and regarding management of overdose

There is no specific antidote for overdose with BRV. Treatment of an overdose should include general supportive measures. Since less than $10 \%$ of BRV is excreted in urine, hemodialysis is not expected to significantly enhance BRV clearance

Clinical trial program study or pivotal article AEs/TEAEs

The most common AEs include sedation, dizziness, nausea, vomiting and rarely changes in behavior may occur. This table includes reviews/summaries of AEs and/or TEAEs - which are reported differently among the studies - see below for breakdown of details.

See clinical trial Table 3, references 4-8, studies marked with symbol " $* \star *$ " were particularly important in defining AE profiles from the clinical trial Table 3. See the text of the article for more detailed discussion specifically of the largest Phase III clinical trial AE/TEAE data (N01358 study, Klein et al. and data available from the clinical trial rosters) [4-8,53]. Pooled postmarketing datasets noted in bibliography $[71,74-76,79,80]$.

AE: Adverse event; AED: Antiepileptic drug; BRV: Brivaracetam; EKG: Electrocardiogram; MD: Physician; PBO: Placebo; POS: Partial-onset seizure; TEAE: Treatment emergent adverse event; UTI: Urinary tract infection. 
Table 5. Adverse events from selected Phase II and III clinical trial data for brivaracetam (cont.).

\section{Safety issue}

French et al. (2010) [48]. Phase Ilb trial, patients enrolled with refractory POS, N001193/NCT00175825

208 patients studied whether or not secondarily generalized. UCB

\section{Detail}

\section{$\mathrm{BRV}$ at $\mathrm{PBO} / 5 / 20 / 50 \mathrm{mg} /$ day}

TEAEs ranged from $52-54 \%$ for all subgroups

There were 54/50/52/52 patients: total 208 patients

Most frequently reported events $>5 \%$ in at least one treatment group

headache/somnolence/influenza/dizziness/neutropenia/fatigue. Of interest, $22 \%$ of patients in PBO group were judged by MDs to have a TEAE likely caused by BRV. Most TEAEs were of mild-to-moderate intensity, severe TEAEs (two in PBO group: 4\%) and two (4\%) in the BRV $5 \mathrm{mg} /$ day group. One patient in the BRV 20 group (2\%) reported serious AEs including somnolence, poor appetite, nausea and ataxia. Few patients permanently discontinued treatment because of TEAEs $2(3.7 \%) / 3(6 \%) / 1(2 \%)$ in the PBO/BRV5/BRV20 groups. Additionally, one patient discontinued in the $50 \mathrm{mg} /$ day group due to pregnancy suspicion

Van Paesschen et al.'s article (2012) [49]. Phase Ilb Placebo (52 patients) BRV $50 \mathrm{mg} /$ day (53 patients) and BRV $150 \mathrm{mg} /$ day (52 patients) Modified from Table 2 in that article:

At least, one TEAE 37 (71\%) in PBO, $36(70 \%)$ in $50 \mathrm{mg} /$ day group $35(67 \%)$ in BRV $150 \mathrm{mg}$ /day group Thought by investigator to be BRV related $22(42 \%)$ - PBO was the same in BRV $50 \mathrm{mg}$ /day group and 19 patients $(37 \%)$ in $150 \mathrm{mg} /$ day group

These included dizziness/headache/nasopharyngitis/nausea/fatigue/somnolence with the highest percentages up to about $15 \%$ and the other complaints of insomnia/anorexia/vomiting/UTI/irritability/back pain/convulsion/attention about $2-6 \%$ of patients generally in various groups, reader is referred to that table for specifics

Ryvlin et al.'s article (2014) [50]. Phase III N01252/NCT004900035

399 patients studied

100 in PBO, 99 in BRV 20 mg/day, 99 in BRV 50 mg/day, 100 in BRV 100 mg/day

Looking at combined BRV groups

There were 181 patients $(61 \%)$ with at least one TEAE, $53(53 \%)$ in PBO group

TEAEs leading to permanent study drug discontinuation four in PBO: $4 \%, 14$ in combined BRV groups $(5 \%)$

Drug-related TEAEs - 31 (31\%) in PBO group, $102(34 \%)$ in the combined BRV groups

Severe TEAEs - $4(4 \%)$ in PBO group, $12(4 \%)$ in the combined BRV group

TEAEs reported by $\geq 5 \%$ in any treatment group included

For combined BRV groups for headache/somnolence/dizziness/fatigue/nasopharyngitis/vertigo/nausea/irritability: $14 / 7 / 6 / 5 / 4 / 4 / 4 / 2 / 2 \%$, respectively

For placebo group: $9 / 6 / 5 / 2 / 1 / 3 / 4 / 0 \%$, respectively

Biton et al.'s article (2014) [51]

Phase III study, 400 patients

Consisted of placebo group, BRV 5, 20 and $50 \mathrm{mg} /$ day

See Table 3 in that article for details

$91 \%$ of patients completed the study and $88 \%$ elected to proceed with the open-label extension study. Most common TEAEs were in the BRV $50 \mathrm{mg} /$ day group, which included somnolence/dizziness/headache/fatigue/insomnia Tracking treatment emergent adverse events $>5 \%$ of patients in any treatment group during the 12-week treatment period

Somnolence/dizziness/headache/fatigue/insomnia/diarrhea/nausea/irritability/influenza/

nasopharyngitis/vomiting/urinary tract infection/upper respiratory tract infection are as follows:

PBO group, $\mathrm{n}=98$ patients, by percentages

$7 / 9 / 14 / 2 / 2 / 2 / 3 / 2 / 1 / 1 / 1 / 2 / 4$

BRV $5 \mathrm{mg} /$ day group, $\mathrm{n}=97$ patients, by percentages

$14 / 12 / 11 / 3 / 2 / 4 / 4 / 2 / 9 / 2 / 3 / 2 / 5$

BRV $20 \mathrm{mg} /$ day group, $\mathrm{n}=100$ patients, by percentages

$14 / 14 / 6 / 13 / 3 / 4 / 7 / 4 / 6 / 4 / 1 / 6 / 5$

BRV $50 \mathrm{mg} /$ day group, $\mathrm{n}=101$ patients, by percentages

$17 / 16 / 13 / 10 / 7 / 6 / 6 / 5 / 4 / 5 / 5 / 1 / 0$

Kwan et al.'s study [52]. Phase III, N01254 study/NCT00504881

359 patients received $\mathrm{BRV}, 121$ received $\mathrm{PBO}$

There were PBO groups as well as BRV $20 \mathrm{mg} /$ day group, $50 \mathrm{mg} /$ day group, $100 \mathrm{mg} /$ day group and $150 \mathrm{mg} / \mathrm{day}$ group.

See Table 1 for AEs in more than $5 \%$ of patients in BRV treatment groups in the 16 -week treatment period There was a dose-finding algorithm in this study, please see the paper for details Serious adverse events included convulsions (3\% in combined BRV groups, $2 \%$ in PBO group) Status epilepticus - three in patient randomized to BRV/none in PBO group One death occurred in the maintenance period - patient received BRV $50 \mathrm{mg} /$ day, drowned while swimming from probable seizure, deemed unlikely from BRV

Patients reporting $\geq 1 \mathrm{AE}$ in BRV-combined groups: $66 \%, 65 \%$ in PBO group Discontinuation due to AEs - $6 \%$ in BRV-combined groups, $5 \%$ in PBO group BRV-combined group and PBO AEs included

Headache/somnolence/dizziness/fatigue/nausea/convulsion/nasopharyngitis/backpain and were $14 / 11 / 9 / 8 / 6 / 5 / 4 / 3 \%$ in BRV-combined groups and $20 / 4 / 6 / 4 / 8 / 3 / 7 / 7 \%$ in the PBO group

Klein et al. (2015) [53]. Phase III study, N01358 study

BRV group contained 503 patients, placebo

TEAEs occurred in $155(59.4 \%)$ of 261 PBO patients versus $340(67.6 \%)$ of 503 BRV-treated patients (safety population) Discontinuation rates due to TEAEs were $3.8,8.3$ and $6.8 \%$ for PBO, BRV $100 \mathrm{mg} /$ day and BRV $200 \mathrm{mg} / \mathrm{day}_{\text {, }}$ respectively

Most frequent TEAEs (PBO vs BRV) were somnolence ( 7.7 vs $18.1 \%$ ), dizziness (5.0 vs $12.3 \%$ ), and fatigue (3.8 vs $9.5 \%$ )

The most common AEs include sedation, dizziness, nausea, vomiting and rarely changes in behavior may occur. This table includes reviews/summaries of AEs and/or TEAEs - which are reported differently among the studies - see below for breakdown of details.

See clinical trial Table 3, references $4-8$, studies marked with symbol "***" were particularly important in defining AE profiles from the clinical trial Table 3 . See the text of the article for more detailed discussion specifically of the largest Phase III clinical trial AE/TEAE data (N01358 study, Klein et al. and data available from the clinical trial rosters) [4-8,53]. Pooled postmarketing datasets noted in bibliography $[71,74-76,79,80]$.

AE: Adverse event; AED: Antiepileptic drug; BRV: Brivaracetam; EKG: Electrocardiogram; MD: Physician; PBO: Placebo; POS: Partial-onset seizure; TEAE: Treatment emergent adverse event; UTI: Urinary tract infection. 
experience in treating epilepsy therefore remains to be fully determined, although some recent publications now exist in the literature [2,16-18,20-22,55-57,59-69].

In the 1960s, several cyclic gamma-aminobutyric (GABA) analogs were made using 2-oxopyrrolidine or GABA moieties with anticipation that such molecules would be GABA agonists that generally cause inhibition of seizures [9,22-24]. Such GABAergic derivative molecules were thought to result in various types of tranquilizers in general and they are also sedative-type compounds $[2,9,23,24]$. This drug development process resulted in piracetam. As the literature indicates, BRV, based on its development, therefore might be considered a 'third-generation offspring' of a lineage of molecules designed to have neuroactive properties, starting with piracetam [9,23,25]. Piracetam was first manufactured in 1964 by Corneliu Giurgea and team who thought the drug might boost mental functioning even in healthy individuals - and it was termed nootropic - a term that was to denote to a senior or former generation of neurologists that such a medication would be a cognitive enhancing agent to describe its properties, and ultimately was launched under the trade name of Nootropil [22-25]. Piracetam was used by Janz in treatment of juvenile myoclonic epilepsy, and this use for this indication by a well-respected clinician after its approval probably gave the moiety some significant clinical credibility [24]. In the USA, piracetam is, however, not approved by the FDA for any medical use and is not permitted to be sold as a dietary supplement. In the UK and Europe, piracetam is prescribed predominantly for myoclonus but also in other conditions as off label use, although large-scale meta reviews (such as those by Cochrane initiative) did not support multiple other indications also postulated such as in dementia or cognitive diagnoses [27].

The antiepileptic properties of LEV were first identified in the 1990s, through screening in audiogenic seizure susceptible mice (1.2). LEV was shown to exhibit saturable and stereospecific binding to a brain-specific-binding site, it did not share with any antiepileptic medication and later studies revealed SV2A to be the molecular correlate to this binding site protein [1-12,22-26]. For a more detailed overview on what receptors/ion channels/proteins that various available antiepileptic medications interact with, please see Table 4 and references.

Review of the basic science and general literature identifies that BRV may have additional features or ramifications compared with LEV [1,2,11-14,19,23,28-35]. For example, BRV in preclinical phase may be superior in rat models of posthypoxic myoclonus and in status epilepticus, although BRV is not currently approved for these indications $[27,28]$. Like other molecules that treat seizures, its mechanism of action inhibits spreading depression in neocortical preparations, and some mechanisms impacted by BRV were speculated to be involved in pain management, although no significant efficacy in the treatment of postherpetic neuralgia has been shown [2,20,27-30]. BRV inhibited epileptiform features in rat hippocampal sections particularly in the CA3 area induced by a high potassium/low calcium perfusion or with addition of bicuculline to baseline perfusion $[23,25,26,28]$. This illustrates a preclinical higher efficacy and potency compared with LEV and uniquely seems to reduce the occurrence of spontaneous epileptiform burst-type discharges within the bicuculline methiodide spikes model, as spikes are inhibited by BRV even at low doses $(\sim 0.1 \mu \mathrm{mol} / \mathrm{l})[1,2,4,11,12,23,25-28,45,47,49]$. Studies have also shown that BRV (unlike LEV) also seems to inhibit seizures induced by maximal electroshock and pentylenetetrazole and shows evidence of protection in self-sustaining status epilepticus in preclinical investigations $[1,2,4,11,12,23,29,44,45,47]$. In corneal-kindled mice, BRV could protect better than LEV against secondarily generalized motor seizures, and pretreatment enhanced those effects [2,28-31]. In hippocampal-kindled rats, similar effects were noted, and in fully amygdala-kindled rats $(50 \mathrm{~Hz}, 1 \mathrm{~s}, 500 \mu \mathrm{A}$ monophasic square wave pulses, once a day, 5 days/week), BRV suppressed seizures and after-discharges nearly completely [2]. In audiogenic seizure-susceptible mice, animals had fewer clonic convulsions induced by auditory stimuli with BRV compared with LEV [2]. Although the current drug approval process sought the indication for POS at this time as noted above, basic research indicates that BRV suppressed significantly spontaneous spike and wave discharges in the Genetic Absence Epilepsy Rat from Strasbourg (GAERS), which serves a model for absence seizures [2]. BRV attenuates generalized photo paroxysmal EEG responses and reverses the inhibitory effects of negative modulators on gamma-aminobutyric acid (GABA) receptors in rat models of status epilepticus $[2,29,31,44,45]$.

\section{Clinical pharmacology of BRV}

$\mathrm{BRV}\{[2 \mathrm{~S}]-2-[(4 \mathrm{R})-2$-oxo-4-propylpyrrolidinyl] butanamide $\}$ is a 2-pyrrolidone derivative $[1,2,9,10,25]$. The discovery and binding site has already been mentioned above $[1,2,9,25]$. BRV has a molecular weight of $212.15 \mathrm{~g} / \mathrm{mol}[1,2,4,7,8]$. The compound is water soluble - having a volume of distribution close to that of total body water at $0.51 / \mathrm{kg}$ [2]. BRV is rapidly bioavailable and nearly completely absorbed after oral administration with linear pharmacokinetics from about 10-600 mg [1,2]. BRV has an elimination half-life of approximately $6-11 \mathrm{~h}$, a geometric mean elimination 
half-life of $9 \mathrm{~h}$, with similar pharmacokinetics and bioavailability in both oral and intravenous (iv.) forms [2,32-37]. $\mathrm{BRV}$ is eliminated mainly by metabolism and less than $10 \%$ by urinary excretion [32-37]. The plasma clearance of $\mathrm{BRV}$ and its metabolites is $0.7-1.3 \mathrm{ml} / \mathrm{min}$ per $\mathrm{kg}$ and the higher clearances are observed in patients that have been studied and comedicated with enzyme inducing AEDs [2,4-8,32-37]. Following repeated administration, at doses of $800 \mathrm{mg} /$ day, there is an increase in metabolic clearance after 2 weeks, this increase in metabolic clearance is not seen in lower doses $\leq 400 \mathrm{mg} /$ day $[2,32-37]$. The blood protein binding is less than approximately $20 \%$. Studies on food interaction show no significant effects [2,31-37]. More than $95 \%$ of an experimental radioactive dose is recovered in the urine within $72 \mathrm{~h}$ after administration [2,31-43]. There are no known active metabolites involving the major metabolic products; a combination of two pathways involving mainly hydrolysis of the acetamide group at the butyramide side chain and through $\omega-1$ hydroxylation of the propyl side chain, mediated by CYP2C19 [35,36]. A recent study indicated that BRV did not significantly impair metabolizing enzymes or transporters, and is unlikely to cause clinically significant drug-drug interactions [43]. BRV pharmacokinetics seems to not be affected by coadministered drugs in general, however, the literature notes some effects with coadministered phenytoin and carbamazepine (CBZ) [37,63,64]. Specifically, in a study by Stockis and Rolan, a Phase I interaction study between BRV and CBZ in 14 healthy subjects showed BRV did not significantly alter CBZ exposure, although it increased the CBZ-epoxide exposure by approximately 2.6-fold and hydrolysis in vitro of the CBZ epoxide was inhibited by BRV, and enzyme induction by CBZ led to only modest reduction in BRV exposure [63,64]. Metabolic reaction phenotyping suggested that BRV disposition potentially is only modestly affected by genetic polymorphism such as in a paper reviewing the influence of the CYP2C19 genotype in Japanese patients [38].

Gender does not seem to alter pharmacokinetics, although bodyweight, use of other enzyme, inducing agents/antiepileptic medications, can cause variability on pharmacokinetics [2,32-37]. Special populations may experience specific pharmacokinetics with BRV such as with the elderly, in populations with impaired renal function, and hepatic impairment and exposures to other medications [32-37,63,64]. In general, it was postulated that these effects do not seem clinically significant enough to effect any significant dose adjustment [32-43]. Impaired hepatic function may result in greater exposure to the medication with elevations in the main alpha metabolite and decrease in the hydroxy metabolite [32-39]. This would make it prudent for clinicians to consider a 50-mg starting dose and a maximal daily dose to $150 \mathrm{mg}$ /day in groups with hepatic impairment [32-39]. Exposure to BRV in patients with liver disease may be increased up to about $60 \%$ and the half-life may increase to about $17 \mathrm{~h}$ in severe liver impairment $[2,32-37,39]$.

BRV's pharmacokinetics that are generally linear over the therapeutic dose range may be altered by enzyme inducers mainly by reducing plasma BRV concentrations up to about $30 \%$ and the half-life may be reduced to about $5 \mathrm{~h}[2,32-37]$. These effects may be mediated by induction of the BRV metabolism with phenytoin, phenobarbital or CBZ (as noted above), although in Phase II data the coadministration of enzyme-inducing AEDs did not significantly change the percentage of responders or worsen seizure frequency, and as already noted - according to the approved product insert labels, no dose adjustments are needed [2,32-37]. It is known that plasma levels may be reduced about $55 \%$ with rifampicin, so in this case, clinicians may consider administering an increased dose of BRV [2,40]. According to UCB data, it seems from pharmacokinetic data that came from some of the clinical trials, BRV up to $200 \mathrm{mg} /$ day did not cause any appreciable changes in plasma concentration of concomitantly administered (seizure medications or oral contraceptives, but only few articles exist and therefore an exhaustive statement on these matters is not clear, see below $[2,37,41-43]$. At high doses of BRV, there may have been up to approximately $15 \%$ reduction in peak serum levels of ethinylestradiol and levonorgestrel in one study but no changes in other hormones indicative of ovulation $[2,37,41,43]$. Exactly what dose or dose titration yields clinical effects in patients is not known, although the clinical trial data indicate that the therapeutic effects may begin at around $50 \mathrm{mg} /$ day [48-53]. At this time, it is noted that BRV interacts with other AEDs as noted by several abstracts and pooled analyses of Phase II/III clinical trial data, along with results from an open-label study without uptitration, including a post hoc analysis evaluating what might predict treatment response $[7,38,48-53,59,60,62]$. Articles indicate that BRV confers no added efficacy or benefit with concomitant LEV [14].

No pharmacokinetic data or effects on pregnant women's exposure are noted, nor are there such data available regarding exposure and or effects in infants who are breastfeeding. BRV is categorized as class $\mathrm{C}$ in pregnancy in the USA $[4,65,66]$. An iv. formulation was studied within the clinical trial program (Table 3 ) $[4,7,8,34,46]$. 


\section{Clinical efficacy of BRV: review of the clinical trial experience}

Although a significant amount of preclinical mechanistic and pharmacokinetic data exist as already noted above, the proposed efficacy of BRV for its therapeutic indication - as with any medication in the USA and EU - is defined mainly through the pivotal Phase IIB and Phase III clinical trials [48-53]. Postmarketing real-world analysis is reviewed below, and discussion from the pooled Phase III dataset will be included in this and subsequent sections. As previously noted, a total of 31 USA- and 18 EU-registered clinical trials exist at present, as previously noted with more than 3000 patients in all, some patients have been in these trials for about 9 years [4-8,46-54]. The complete set of clinical trial designations and significance and correspondence to UCB protocol designation and clinical trial numbers at the FDA and EU EMA and resultant peer review or other publications and significance are summarized in Tables 3 and 4 from information currently available in the public domain. These tables aim to cross-reference clinical trial numbers in the EU and USA, UCB protocols, publications and other data in an organized compendium.

This section of the manuscript will discuss the relevant summary of the Phase IIb and pivotal Phase III studies highlighting the clinical efficacy of BRV in the clinical development program. This section will discuss the initial disappointment from Phase II and early Phase III trials in the trial experience and the resolution that followed. But after this initial disappointment, ultimately the entire compendium of BRV's Phase III clinical trial program that resulted over this time period from approximately 2007 until present is what ultimately led to the approval of BRV.

For all of the clinical trials, the primary end point for the European regulatory authorities is the $50 \%$ responder rate for POS frequency compared with placebo, over the treatment period standardized to the 28-day duration [5]. The primary end point for the FDA is the percent reduction compared with placebo for POS frequency, over the treatment period standardized to the 28-day duration [6]. During the clinical trial periods prior to approval, both a Phase II and a Phase III trial had issues of not meeting their primary end points, see below for discussion causing administrative hurdles at the FDA and EMA that assisted in development of the later clinical trials [47-49]. In retrospect, it seemed that these trials likely failed to reach the proposed end points mainly because of the hierarchical statistical design that included detailed analysis of dosing BRV less than $50 \mathrm{mg} /$ day, which further analysis over multiple studies identifies that these doses are simply not efficacious when using BRV for seizure control, see below for details [47-49].

The pivotal studies performed in the Phase III program ultimately consisted of studies designated as UCB protocols N01252, N01253, N01254, and N01358 (Table 4 \&5) [48-53]. These protocols corresponded to clinical trials in the EU and USA and ultimately led to various peer-reviewed publications, see below and the corresponding designations in Tables $3 \& 4$. These studies reviewed the safety and efficacy and tolerability of adjunctive BRV from 20 to $200 \mathrm{mg} /$ day (see Tables 1-5) [48-53]. Patients in these four study protocols included patients with or without POS that secondarily generalized and there were also refractory patients with POS included in subgroups containing more than 1 but up to and beyond three AEDs in some cases.

The most recent study performed prior to approval in the EU and USA is the N01358 study. published by Klein et al. in the latter portion of 2015 [53]. This study aimed to confirm the safety and efficacy and safety and tolerability in POS from ages 16 to 80 years. This is one of the largest Phase III studies to date and excluded patients on LEV. BRV was added without a dose finding uptitration at a starting dose. 768 patients were studied with BRV dosing up to $200 \mathrm{mg} /$ day. French $e t$ al. published results of an exploratory dose-finding study that reflected a promising overview of efficacy and tolerability data, but in retrospect after this trial it seemed that the doses up to $50 \mathrm{mg}$ /day were likely subtherapeutic, and in subsequent studies (see below) higher doses met efficacy end points in the collective compendium in the later Phase III study program [48,49,54].

An additional study as noted by Van Paesschen et al. - a Phase IIB dose-finding study was performed that investigated doses from 50 to $150 \mathrm{mg} /$ day in a total of 157 patients, with approximately 50 patients per treatment arm (1:1:1 to placebo, BRV 50 or $150 \mathrm{mg}$ /day) [49]. These exploratory dose-finding studies indicated that BRV at $50 \mathrm{mg} /$ day might be a starting dose and extended the range of administration to $200 \mathrm{mg} /$ day, although in the Van Paesschen et al.'s study, no difference was seen between the 50 and $150 \mathrm{mg}$ daily doses of BRV [48,49]. Additionally as already alluded to, study N01252 which was considered a pivotal study, did not meet statistical significant for the $50 \mathrm{mg}$ dose (its primary efficacy end point) based on a predefined sequential testing strategy, as noted by Ryvlin $e t$ al. [4-8,50]. The primary efficacy analysis (percent reduction over placebo in baseline-adjusted focal seizure frequency per week) based on the $50 \mathrm{mg} /$ day dose was not statistically significant. However, the primary efficacy end point 
for the BRV $100 \mathrm{mg} /$ day arm did achieve statistical significance $(\mathrm{p}=0.037)$. Secondary efficacy analyses provided supportive evidence for the efficacy of $100 \mathrm{mg}$ /day [50].

The subsequent pivotal study (N01253) examined doses of 5, 20 and $50 \mathrm{mg} /$ day and confirmed that the $50 \mathrm{mg} /$ day dose shows statistically significant efficacy as outlined by Biton et al. [4-8,51,48-52]. In a Phase III, double-blind, randomized, placebo-controlled, flexible-dose trial, Kwan et al. reported that individualized tailored doses $(20-150 \mathrm{mg} /$ day) were well tolerated in adults with uncontrolled epilepsy, and that the results provide support for further evaluation of efficacy in reducing focal and generalized seizures [52]. It seems that a pooled analysis of three fixed dose, Phase III studies showed statistically significant median percentage reduction in both weekly focal seizure frequency from baseline and in general, with BRV there is a $\geq 50 \%$ responder rate for BRV compared with placebo starting at $50 \mathrm{mg} /$ day (Table $3 \& 4$ ) [46-53]. Results from the N01358 study indicate that the percent reduction over placebo also occurred with statistical significance within the design protocol of patients receiving doses of BRV up to $200 \mathrm{mg} /$ day [53]. Although the clinical trial program met end points for the control for epilepsy, studies of BRV in patients with Unverricht-Lundborg disease with progressive myoclonic epilepsy did not provide statistically significant clinical benefit in reaching the primary end point of reducing the severity of action myoclonus (as measured by the Unified Myoclonus Rating Scale), however, this may have been due to the high variability in disease severity as well as the lower doses that were used $(2.5-50 \mathrm{mg} /$ day) [4-8,61]. Additionally these were done earlier than the Phase III studies for BRV at a period in time, when it was not proven that such low doses of BRV might not have appreciable efficacy in POS, but in the subsequent publication in 2016, BRV up to $150 \mathrm{mg} /$ day was studied and was deemed well tolerated, but primary end point of statistically significant control of myoclonus was not demonstrated [61]. It was speculated that rare subpopulations of various epilepsy populations might require yet to be defined methods of study for demonstrating optimal indication [61].

Additionally, two international conversion to monotherapy BRV studies (N01276 and N01306) were conducted but were halted as a review by an independent data monitoring committee determined that predefined exit criteria for stopping the studies were met, but no unexpected safety concerns were noted [2,4-8,14]. In retrospect from review of the clinical trial program, the disappointing failure of the early clinical trials for BRV for meeting end points in the Phase II/III program was likely because of including doses less than $50 \mathrm{mg} /$ day in the overall analyses and not due to the study design of these types of clinical trials, and the reader is referred to the trials that settled this issue and literature reviewing the general study design for AED trials [49-54].

\section{Safety \& tolerability: results \& AEs from Phase III studies}

The most frequent TEAEs, in general, seem consistent across the large Phase III study program and were somnolence, dizziness and fatigue, as noted in Klein et al. [53]. This article by Klein et al. is one of the largest Phase III trials completed on BRV just prior to and at the perilaunch period of BRV [53]. However, additional studies have appeared later and postmarketing such as those from Ben-Manachem et al., Kalvianen et al., Lattanzi et al., Toledo et al. and Asadi-Pooya et al. [59,61,62,75,76]. This section will review the preapproval dataset, please see the Table 5 regarding TEAEs/SAEs that will also review the known complete pooled dataset at time of this article's publication that also includes data from the ongoing clinical trials and the above.

Klein $e$ al. indicate that TEAEs occurred in 155 (59.4\%) of $261 \mathrm{PBO}$ patients and in $340(67.6 \%)$ of 503 BRV-treated patients in that article [53]. Klein et al. note that the discontinuation rates due to TEAEs were 3.8, 8.3 and $6.8 \%$ for PBO, BRV $(100 \mathrm{mg} /$ day $)$ and BRV $(200 \mathrm{mg} /$ day $)$, respectively [48]. Most frequent TEAEs ( $\mathrm{PBO}$ vs BRV) were somnolence (7.7 vs $18.1 \%$ ), dizziness (5.0 vs $12.3 \%$ ) and fatigue (3.8 vs 9.5\%) [53]. Study discontinuation rates for any reason were 11 and $10 \%$ for BRV at 100 and $200 \mathrm{mg} /$ day versus $7 \%$ for placebo were previous statements released from the UCB website from data on file as part of this trial [53]. Headache in placebo and BRV groups' percentage was similar at approximately $8 \%$. BRV group contained 503 patients, placebo group contained 261 patients [53].

Additionally, $88 \%$ of patients in the N01358 trial elected to go into the open-label phase and receive BRV. In subgroup analysis, it was also noted that seizure reduction occurred in those that had previously discontinued off LEV as well as patients that were not previously exposed to LEV [53]. This efficacy in patients with prior LEV or other AED exposure was confirmed in another post hoc analysis [78]. TEAEs, in general, were more common in the BRV-treated patients compared with placebo, with the majority of such being mild or moderate, see Table 5 and figures below [48]. Psychiatric TEAEs were noted also to be mild or infrequent, and included anxiety $(1.1 \%$ placebo; $2.2 \%$ BRV overall), insomnia (1.1\% placebo; $2.0 \%$ BRV overall) and depression ( $0.4 \%$ placebo; $0.8 \%$ BRV overall) [53]. The overall incidence of psychiatric TEAEs was similar for both BRV dosages in that study 
$(10.3 \% 100 \mathrm{mg} /$ day and $11.2 \% 200 \mathrm{mg} /$ day) versus placebo (7.7\%) [53]. The incidence of suicidal ideation was low and equal for placebo, two deaths occurred during that study; one was attributed to sudden unexplained death in epilepsy patients (SUDEP), and the other was by unknown mechanism, but both thought not due to BRV [53].

In general, it seems that pooled data from the studies by French et al., Van Paesschen et al., Ryvlin et al., Biton et al. and Kwan et al.'s studies - the noted AEs or TEAEs (sometimes reported either way in the studies but representing the same designation) profile seems consistent throughout the Phase II and III trials [48-52]. AEs include nausea, vomiting, fatigue, anorexia, dizziness, headache, somnolence, insomnia, nasopharyngitis [48-52]. They were typically mild to moderate in severity, generally similar to that of the Klein et al.'s study cited above [48-53]. Please see Table 5 for more exact breakdown of these items. Nonpsychotic behavioral adverse events (BAEs) with LEV and BRV are of particular concern because previous studies have shown about a $13 \%$ of patients treated with LEV reported a behavioral symptom compared with about $6 \%$ that with a placebo [48-53]. Articles also identify that it has been suggested that patients taking LEV clinically experience behavioral events above that which is reported in the clinical trials, which may impact long-term tolerability $[19,55,56]$. The emergence of side effects or behavioral effects including suicide seemed to be a reason for discontinuing LEV [19,53,55,56]. About $54 \%$ of patients continued LEV as adjunctive therapy for POS after 2 years, whereas $46 \%$ had discontinued, and BAEs contributed to about $40 \%$ of the cases who had discontinued therapy [56].

Yates et al.'s recent review of NCT01653262 Phase IIIb study noted that of the 29 patients enrolled, 26 (89.7\%) completed the study and at the conclusion of the treatment period, 27/29 (93.1\%) patients switched to BRV had clinically meaningful reductions in BAEs [19]. Yates et al. studied evaluated BAEs in patients receiving LEV, who switched to BRV [19]. Patients $\geq 16$ years of age receiving 2-3 AEDs including LEV at $1-3 \mathrm{~g} /$ day, and experiencing BAEs within 16 weeks of LEV initiation enrolled in this Phase IIIb open-label study with a less than 1-week screening period, and an immediate switch from LEV to BRV at $200 \mathrm{mg}$ /day without a titration and a 12-week treatment period and had Quality of Life in Epilepsy Inventory - Form 31 (QOLIE-31-P) scales performed [19]. Of the 29 patients enrolled, 26 (90\%) completed the study and at the end of the treating period, 27/29 (93\%) switched to BRV and had meaningful reductions in the BAEs [19]. Physician reports of BAEs also reported reductions in BAEs in $93 \%$ of patients. 23/29 patients reported TEAEs with one patient having reported a suicidal ideation and a suicidal attempt [19]. Two patients discontinued BRV during the study. Of the two patients that discontinued BRV, one discontinued due to perceived lack of efficacy and the other reported a suicidal ideation and attempt [19]. Nonbehavioral TEAEs were reported in $23 / 29$ patients (79\%) included headache $-5 / 29$ patients (17.2\%), fatigue - three patients (10.3\%) and back pain - three patients (10.3\%). Additional TEAEs that were reported included depression, dizziness, insomnia, nasopharyngitis and tremor, each of these in two patients at 7\% [19].

In the Biton et al.'s study of 2014, a noted reason for discontinuing BRV was behavioral abnormalities and according to Pack, it seems that comparing nonpsychotic BEAs such as irritability and aggression, the numbers/percentages reported may be less than that of LEV, but since no head-to-head comparison has been done at the time of the Pack's article publication, the recommendation at that time was noted that any such claim should be viewed circumspectly [14,51]. In the Ryvlin et al., Kwan et al., French et al. and Van Paesschen et al.'s studies, prior LEV exposure may be associated with an attenuated effect of BRV [48-50,52,78]. Table 5 reviews comparative study designs, cohorts of subcategories of patients and corresponding AEs or TEAEs in further detail.

Preclinical studies mentioned that the maximal nonlethal oral dose is above $1000 \mathrm{mg} / \mathrm{kg}$ with no effects to about $500 \mathrm{mg} / \mathrm{kg}$ in animal studies $[1,2,4-8]$. For additional discussion and more comprehensive review of AEs/TEAEs, please see Table 5. A review of comparative neurocognitive effects of BRV and LEV and lorazepam is noted in the literature [57].

\section{Postmarketing surveillance \& recent newer developments peri- to postlaunch of BRV}

The postmarketing phase began after the approval and launch of BRV and the reader is referred to publications that document that process and articles reviewing the preclinical properties of BRV and BRV development are now available [65-83]. Review of the EU and US clinical trial rosters shows that there are multiple clinical trials that are currently continuing into the postmarketing phase (Table 3) [4-8]. There have been pre- and postmarketing abstract presentations at the American Academy of Neurology in 2016 and 2017 and at the American Epilepsy Society meetings in 2015 and 2016 noting newer developments and observations [68,69]. For example, data delineating the enhanced brain permeability for BRV as compared with LEV were noted in these meetings, and some of these abstracts and or related concepts more recently are now published [68,69,73,74]. In 2017, at the American Academy of Neurology, Moseley et al. also reviewed the drug-drug interactions of BRV in the peri- to postmarketing phase 
and identified the efficacy of adjunctive BRV in patients with secondarily generalized tonic-clonic seizures at baseline using pooled results from long-term follow-up studies, and this is now published [75]. Evaluation of abuse potential of BRV in healthy recreational CNS-depressant users is being explored and defined [66-69]. Pooled analysis of the three pivotal clinical studies are now available $[59,60,62]$. Newer data identified during the perilaunch period regarding pharmacokinetics BRV when taken with other medications are noted in the recent literature [63,64,67]. Steinig et al. published a postmarketing trial in Germany and Milovanocic et al. provide an overview at time of launch of BRV [70,71]. Steinig et al.'s study noted that 262 patients were treated with BRV from 1 day to 12 months with a median retention time of 6.1 months and identify that the retention rate at 3 months was 79.4 and $75.8 \%$ at 6 months [70]. $14.9 \%$ were seizure free at 3 and 6 months, $15.3 \%$ were seizure free in the Steinig et al.'s study [71]. TEAEs were observed in $37.8 \%$ with the most common being somnolence, dizziness and BAEs. BAEs that presented under previous LEV treatment improved upon switch to BRV in 57.1\% (20/35 patients) and LEV-induced somnolence improved in 70.8\% (17/24 patients) [70]. Patients with BAEs on LEV were, in general, seemed more likely to develop BAE(s) on BRV, odds ratio: 3.48, 95\% CI: 1.53-7.95 [70].

More recently, Russo et al. opined that the increased permeability of BRV compared with LEV may be useful in emergency treatment for seizures, although there is currently no approved indication for this [67]. Subsequently, the postmarketing literature notes a potential role of BRV in both uncontrolled POS and in status epilepticus [67,79-82]. Specifically recent postmarketing studies note efficacy in refractory uncontrolled POS and in status epilepticus and possibly super-refractory status epilepticus [79-82]. Experimental models in animal models may indicate enhanced CNS drug entry time of 3 min for BRV compared with $23 \mathrm{~min}$ for LEV, which represents a factor of about seven-fold increase in entry speed into the brain [74]. Further publications of postmarketing meta-analyses such as by Brigo et al. and Zhu et al. involving comparing BRV to multiple products and adjunctive medications available for POS are also noted in the recent literature [81,82].

In general, at this point in time, physicians are pondering as in the 2017 French commentary and replies and as in this current paper - how exactly BRV will impact their epilepsy patients based on the preclinical experience, the clinical trial program and the recent abstracts, presentations and publications of the postmarketing literature [83,84].

\section{Conclusion}

BRV underwent an initial complex research and development followed by a complex series of Phase III investigations starting around 2007 that ultimately led to approval by the EMA and the subsequent launch in the UK. Subsequently, BRV was approved for use by the FDA in February 2016. The FDA approved BRV for treatment of POS in monotherapy in patients $\geq 16$ years old in September 2017 as a unique antiseizure medication without a dosefinding uptitration.

This article speculates that BRV has the potential to make a significant impact on the treatment of epilepsy from the review of the current clinical trial program and the initial and early approximately 18-month postmarketing experience. The clinical trial program included a representative cohort of some of the most difficult to manage patients in clinical practice including those with secondarily generalized seizures, and in refractory patients that were on adjunctive 1-3 or more additional agents for POS. Data to date seem to indicate an acceptable side-effect profile and while the full extent of BAEs are not known with agents that interface with the SV2A mechanism, recent publications of small cohorts of patients who had previously experienced BAEs on LEV suggest that patients might benefit by switching from LEV to BRV. Initial Phase IIb trials and one of the pivotal Phase III trials were disappointing as they did not meet statistical significance for the primary end point, which prompted discussions with and guidance from the FDA and EU, which led to a redesign on the Phase III program that was ultimately successful. Additional studies were performed that showed statistically significant efficacy for the respective primary end points. The initial disappointing performance of BRV in the clinical trials was likely due to studying total daily doses less than $50 \mathrm{mg}$ and not due to the study design of the clinical trial program itself. After studies were designed with dosing from 50 to $200 \mathrm{mg} /$ day, the Phase III program met statistical end points.

Exactly how BRV will have an impact in treating epilepsy within a marketplace with multiple options, remains to be fully determined. Postmarketing analyses continue that will potentially clarify BRV's role in treating epilepsy. As of the time of the publication of this article effective doses in clinical trials as noted above have been studied from the range of 50-200 mg/day. BRV as compared with LEV has an enhanced mechanism of action at the molecular target. BRV exhibits more rapid brain permeability presumably due to a different chemical structure compared with LEV, and preliminarily there is a different and potentially more favorable potential BEA profile compared with LEV, the full extent may ultimately be delineated in the evolving postmarketing literature. There 
may also be additional efficacy in patients previously not gaining efficacy with LEV or other currently available medications in a market place that contains a significant epidemiology patients with refractory epilepsy despite the already numerous treatment and products available. The preclinical and animal models outline a potentially enhanced and different antiepileptic effect profile for BRV mechanistically as compared with LEV. In general to date, the clinical trial program along with select pooled and postmarketing datasets indicate an overall acceptable side-effect profile. Oral tablets, an iv. formulation and an oral solution were all investigated within the clinical trial program. As this review suggests, it is anticipated therefore that BRV represents a potentially successful option in treating epilepsy.

\section{Executive summary}

\section{Overview of brivaracetam}

- Brivaracetam (BRV) developed and marketed by UCB Pharma, 'third-generation molecule' from piracetam ('first generation'; 1960s), which the development program yielded levetiracetam (LEV; 'second generation; C. 1992) and now BRV ('third generation').

- Multiple clinical trials: approximately 3000 patients in 31 USA and 18 EU trials with including refractory epilepsy patients (those patients included were on one to three or more antiepileptic drugs, and some patients experienced secondarily generalized seizures, and others on LEV vs being LEV naive) some having an approximate 9 -year experience in the trials.

- Tablet, oral solution and intravenous forms exist.

Mechanism of action

- BRV is a novel SV2A ligand, chemically related to LEV. BRV binds SV2A with ten- to 30-fold higher affinity compared with LEV specificity for SV2A about 30-times more compared with LEV.

- BRV inhibits epileptiform discharges and in the available animal models and may offer neural protection in epilepsy compared with LEV.

- Unlike LEV, BRV has activity protecting cells from becoming epileptogenic or attenuates epileptiform discharges in maximal electroshock models, corneal kindled mice and hippocampal kindled animals in preclinical models.

- In audiogenic seizure susceptible mice, BRV prevents more convulsive motor seizures compared with LEV.

- BRV suppresses epileptiform discharges more completely in Generalized Auditory Epilepsy Rats of Strasbourg models.

- BRV and LEV when taken together seem to not have any added benefit.

Safety \& tolerability

- Current data indicate: somnolence, dizziness, fatigue and headache (which seems, however, in some of the Phase III trials to occur at similar rate as placebo-treated patients) are the most common adverse effects. Nausea and anorexia have also been noted, see Discussion and Table 5 for details.

- Discontinuation of BRV in clinical trials for any reason: $11 \%$ (compared with $7 \%$ in the placebo group).

- Treatment emergent side effects for any issue in the recent clinical trial (NCT01261325) and pooled data: 100 and $200 \mathrm{mg} /$ day groups were 68 and $67 \%$, respectively, and around $59 \%$ in placebo control group.

- BRV may represent a potential option for patients who have previously failed LEV.

Financial \& competing interests disclosure

The author was principal investigator (PI) on a number of Clinical Trials, including with UCB, had been consultant speaker previously but not after 2009. The author has no other relevant affiliations or financial involvement with any organization or entity with a financial interest in or financial conflict with the subject matter or materials discussed in the manuscript apart from those disclosed.

No writing assistance was utilized in the production of this manuscript.

\section{Company review}

In addition to the peer-review process, with the author's consent, the manufacturer of the product discussed in this article was given the opportunity to review the manuscript for factual accuracy. Changes were made by the author at their discretion and based on scientific or editorial merit only. The author maintained full control over the manuscript, including content, wording and conclusions. 


\section{Open access}

This work is licensed under the Creative Commons Attribution-NonCommercial-NoDerivatives 4.0 Unported License. To view a copy of this license, visit http://creativecommons.org/licenses/by-nc-nd/4.0/

\section{References}

Papers of special note have been highlighted as: • of interest; $\bullet \bullet$ of considerable interest

1. Von Rosenstiel P. Brivaracetam (UCB 34717). Neurotherapeutics 4, 84-87 (2007).

- It overviews the preclinical properties of brivaracetam (BRV) as UCB transitions to the clinical trial program.

2. Von Rosenstiel P, Perucca E. Chapter 35: brivaracetam. In: The Treatment of Epilepsy (3rd Edition). Shorvon SD, Perucca E, Engel J (Eds). Wiley-Blackwell, NJ, USA, 447-457 (2009).

3. Rogawski MA. Brivaracetam: a rational drug discovery success story. Br. J. Pharmacol. 154, 1555-1557 (2008).

4. UCB Pharma. www.ucb.com

5. Search of studies: brivaracetam. https://clinicaltrials.gov/ct2/results?term=brivaracetam\&pg=1

6. Search clinical trials: brivaracetam. www.clinicaltrialsregister.eu/ctr-search/search?query=Brivaracetam

7. Briviact US Prescribing Information. UCB, Brussels, Belgium (2017). www.briviact.com/briviact-PI.pdf

8. Briviact EU Prescribing Information. UCB, Brussels, Belgium (2016). www.ema.europa.eu/docs/en_GB/document_library/EPAR_-_Product_Information/human/003898/WC500200206.pdf

9. Kenda BM, Matagne AC, Talaga PE et al. Discovery of 4-substituted pyrrolindone butanamides as new agents with significant antiepileptic activity. J. Med. Chem. 47, 530-549 (2004).

10. Lynch BA, Lambeng N, Nocka $\mathrm{K}$ et al. The synaptic vesicle protein SV2A is the binding site for the antiepileptic drug levetiracetam. Proc. Natl Acad. Sci. USA 101, 9861-9866 (2004).

11. Matagne A, Margineanu D, Kenda B et al. Anti-convulsive and anti-epileptic properties of brivaracetam (UCB 24714), a high affinity ligand for the synaptic vesicle protein, SV2A. Br. J. Pharmacol. 154, 1662-1671 (2008).

- Identifies the mechanism of action of BRV and is of interest.

12. Zona C, Pieri M, Carunchio I et al. Brivaracetam (ucb 34714), a high affinity ligand for the synaptic vesicle protein, SV2A. Br. J. Pharmacol. 154, 1662-1671 (2008).

13. Mercier J, Holden D, Deo AK et al. Brivaracetam achieves brain SV2A occupancy faster than levetiracetam. Epilepsy Curr. 15(Suppl. 1), 343 (2015).

14. Pack AM. Brivaracetam, a novel antiepileptic drug: is it effective and safe? Results from one Phase III randomized trial. Epilepsy Curr. 14(4), 196-198 (2014).

15. Gillard M, Fuks B, Leclerq K et al. Binding characteristics of brivaracetam, a selective, high affinity SV2A ligand in rat, mouse, and human brain: relationship to anti-convulsant properties. Eur. J. Pharmacol. 664, 36-44 (2011).

16. Hauser WA, Annegers JF, Kurland LT. Prevalence of epilepsy in Rochester, MN, 1940-1980. Epilepsia 32, 429-445 (1991).

17. Thurman DJ, Beghi E, Begley CE et al. ILAE Commission on Epidemiology. Standards for epidemiologic studies and surveillance of epilepsy. Epilepsia 52(Suppl. 7), 2-26 (2011).

18. Epilepsy across the spectrum: promoting health and understanding (2012). http://iom.nationalacademies.org/Reports/2012/Epilepsy-Across-the-Spectrum.aspx\#sthash.1w0ZE4E5.dpuf

19. Yates SL, Fakhoury T, Liang W et al. An open-label, prospective, exploratory study of patients with epilepsy switching from levetiracetam to brivaracetam. Epilepsy Behav. 52, 165-168 (2015).

- It is the earliest publication available on patients who may have fewer behavioral side effects with BRV as compared with levetiracetam.

20. Wiebe S, Blume WT, Girvin GT et al. A randomized, controlled trial of surgery for temporal-lobe epilepsy. N. Engl. J. Med. 345, 311-318 (2001).

21. Jobst BC, Cascino G. Resective epilepsy surgery for drug-resistant focal epilepsy. JAMA 313(3), 285-293 (2015).

22. von Rosenstiel P, Perucca E. Chapter 35: Brivaracetam. In: The Treatment of Epilepsy (3rd Edition). Shorvon SD, Perucca E, Engel J (Eds). Wiley-Blackwell, NJ, USA (2009).

23. Zona C, Pieri M, Klitgaard H et al. Ucb 34717, a new pyrolidone derivative, inhibits Na+-currents in rat cortical neurons in culture. Epilepsia 45(Suppl. 7), 146 (2004).

24. Gouliaev AH, Senning A. Piracetam and other structurally related nootropics. Brain Res. Rev. 19(2), 180-222 (1994).

25. Margineanu DG, Kenda B, Michel P et al. Ucb 24717, a new pyrrolidone derivative: comparison with levetiracetam in hippocampal slice epilepsy models in vitro. Epilepsia 44(Suppl. 9), 261 (2003). 
26. Shorvon SD. Chapter 48: Piracetam. In: The Treatment of Epilepsy (3rd Edition). Shorvon S, Fish D, Perucca E. (Eds). Wiley-Blackwell, NJ, USA, 489-495 (2004).

27. Zhou Q, Hu CY, Zhang W, Huang YH. Brivaracetam add-on therapy for epilepsy (Protocol). Cochrane Database Syst. Rev. (2015). http://onlinelibrary.wiley.com/doi/10.1002/14651858.CD011501/epdf

28. Tai KK, Truong DD. Brivaracetam is superior to levetiracetam in a rat model of post-hypoxic myoclonus. J. Neural. Transm. 114, 1547-1551 (2007).

29. Wasterlain C, Suchomelova L, Matagne A et al. Brivaracetam is a potent anticonvulsant in experimental status epilepticus. Epilepsia 46, 219-220 (2005).

30. Lamberty Y, Ardid D, Eschalier A et al. A new pyrrolidone derivative UCB 24717 is effective in neuropathic pain models in rats: comparison with gabapentin. J. Pain 4(Suppl.), 53 (2003).

31. Margineanu DG, Klitgaard H. Brivaracetam inhibits spreading depression in rat neocortical slices in vitro. Seizure 18, 453-456 (2009).

32. Sargentini-Maier ML, Rolan P, Connell J et al. The pharmacokinetics, CNS pharmacodynamics and adverse event profile of brivaracetam after single increasing oral doses in healthy males. Br. J. Clin. Pharmacol. 63(6), 680-688 (2007).

33. Rolan P, Sargentini-Maier ML, Pigeolet E et al. The pharmacokinetics, CNS pharmacodynamics and adverse event profile of brivaracetam after multiple increasing oral doses in healthy males. Br. J. Clin. Pharmacol. 66, 71-75 (2008).

34. Hulhoven R, Scheen A, Watanabe J et al. Bioavailability and safety of the intravenous administration of brivaracetam. Epilepsia 49(Suppl. 7), 438 (2008).

35. Sargentini-Maier ML, Espie P, Coquette A et al. Pharmacokinetics and metabolism of 14C-brivaracetam, a novel SV2A ligand in healthy subjects. Drug Metab. Dispos. 36, 36-45 (2008).

36. Sargentini-Maier ML, Sokalski A, Boulanger P et al. Brivaracetam disposition in renal impairment. J. Clin. Pharmacol. 52, 1927-1933 (2012).

37. LaCroix B, Rosenstiel P, Sargintini-Maier M-L. Population pharmacokinetics of brivaracetam in patients with partial epilepsy. Epilepsia 48(Suppl. 6), 333 (2007).

38. Stockis A, Watanabe S, Rouits E et al. Brivaracetam single and multiple rising oral dose study in healthy Japanese participants: influence of CYP2C19 Genotype. Drug Metab. Pharmacokinet. 29, 394-399 (2014).

39. Stockis A, Sargentini-Maier ML, Horsmans Y. Brivaracetam, disposition in mild to severe hepatic impairment. J. Clin. Pharmacol. 53(6), 633-641 (2013).

40. Stockis A, Wantanabe $S$, Sheen A et al. Effect of rifampin on the disposition of brivaracetam in human subjects: further insights into brivaracetam hydrolysis. Drug Metab. Dispos. 44, 792-799 (2016).

41. Stockis A, Watanabe S, Fauchoux N. Interaction between brivaracetam $(100 \mathrm{mg} /$ day $)$ and a combination oral contraceptive: a randomized, double-blind, placebo-controlled study. Epilepsia 55(3), e27-e31 (2014).

42. Shoemaker R, Wade J, Stockis A. Brivaracetam population pharmacokinetics and exposure-response modeling in adult subjects with partial onset seizures. J. Clin. Pharmacol. 56(12), 1591-1602 (2016).

43. Chanteux H, Kervyn S, Gerin B et al. In vitro pharmacokinetic profile of brivaracetam (BRV) reveals low risk of drug-drug interaction (DDI) and unrestricted brain permeability (P4.276). Neurology 84(Suppl. 14) (2015).

44. Kasteleijn-Nolst T, Genton P, Parain D et al. Evaluation of brivaracetam, a novel SV2A ligand in the photosensitivity model. Neurology 69, 1027-1034 (2007).

45. Wasterlain GC, Suchomelova L, Matagne A et al. Brivaracetam is a potent anticonvulsant in experimental status epilepticus. Epilepsia 46(Suppl. 8), 219 (2005).

- Reviewing BRV's preclinical theoretical utility in status epilepticus.

46. Klein P, Biton V, Dilley D et al. Safety/tolerability of adjunctive intravenous brivaracetam as infusion of bolus in patients with epilepsy. Epilepsy Curr. 14(Suppl. 1), 389 (2014).

47. Perucca E. What is the promise of new antiepileptic drugs in status epilepticus? Focus on brivaracetam, carisbamate, lacosamide, NS-1209, and topiramate. Epilepsia 50(Suppl. 12), 49-50 (2009).

48. French JA, Costantini C, Brodsky A et al. Adjunctive brivaracetam for refractory partial-onset seizures: a randomized, controlled trial. Neurology 75(6), 519-525 (2010).

49. Van Paesschen W, Hirsch E, Johnson $\mathrm{M}$ et al. Efficacy and tolerability of adjunctive brivaracetam in adults with uncontrolled partial-onset seizures: a Phase IIb, randomized, controlled trial. Epilepsia 54(1), 89-97 (2013).

50. Ryvlin P, Erhahn KJ, Blaszcyck B et al. Adjunctive brivaracetam in adults with uncontrolled focal epilepsy: results from a double-blind, randomized, placebo-controlled trial. Epilepsia 55(1), 47-56 (2014).

51. Biton V, Berkovic SF, Abou-Khalil B et al. Brivaracetam as adjunctive treatment for uncontrolled partial epilepsy in adults: a Phase III randomized, double blind, placebo controlled trial. Epilepsia 55(1), 57-66 (2014).

52. Kwan P, Trinka E, Van Paesschen W et al. Adjunctive brivaracetam for uncontrolled focal and generalized epilepsies: results of a Phase III double-blind, randomized, placebo-controlled, flexible-dose trial. Epilepsia 55(1), 38-46 (2014). 
53. Klein P, Schiemann J, Sperling MR et al. A randomized, double-blind, placebo-controlled, multicenter, parallel-group study to evaluate the efficacy and safety of adjunctive brivaracetam in adult patients with uncontrolled partial-onset seizures. Epilepsia 56(12), 1890-1898 (2015).

-• Represented the largest overview of the premarketing clinical efficacy and safety data.

54. French JA, Wang S, Warnock B, Tem N. Historical control monotherapy design in the treatment of epilepsy. Epilepsia 51(10), 1936-1943 (2010).

55. Yates PI, French J, Edrich P et al. A systematic review of the safety profile of levetiracetam: a new antiepileptic drug. Epilepsy Res. 47 , 77-90 (2001).

56. Chung S, Wang N, Hank N. Comparative retention rates and long-term tolerability of new antiepileptic drugs. Seizure 16, 296-304 (2007).

57. Meador KJ, Gevins A, Leese PT et al. Neurocognitive effects of brivaracetam, levetiracetam, and lorazepam. Epilepsia 52(2), 264-272 (2011).

58. Rogawski MA. A new SV2A ligand for epilepsy. Cell 167, 587 (2016).

-• Outlines the current molecular properties of the SV2A receptor.

59. Ben-Menachem E, Mameniškienė R, Quarato PP et al. Efficacy and safety of brivaracetam for partial-onset seizures in 3 pooled clinical studies. Neurology 87(3), 314-323 (2016).

60. Markham A. Brivaracetam: first global approval. Drugs 76(4), 517-522 (2016).

61. Kälviäinen R, Genton P, Andermann E et al. Brivaracetam in Unverricht-Lundborg disease (EPM1): results from two randomized, double-blind, placebo-controlled studies. Epilepsia 57(2), 210-221 (2016).

62. Lattanzi S, Cagnetti C, Foschi N et al. Brivaracetam add-on for partial-onset seizures in 3 pooled clinical studies. Neurology 86(14), 1344-1352 (2016).

63. Stockis A, Rolan P. Brivaracetam and carbamazepine interaction in healthy subjects and in vitro. Epilepsy Res. (113), 19-27 (2015).

64. Stockis A, Brodie $\mathrm{M}$ et al. Pharmacokinetic interaction of brivaracetam on carbamazepine in adult patients with epilepsy, with and without valproate co-administration. Epilepsy Res. (128), 163-168 (2016).

65. Source: US FDA. FDA approves briviact to treat partial onset seizures. Press release: www.fda.gov/newsevents/newsroom/pressannouncements/ucm 486827.htm

66. Drug Enforcement Administration Department of Justice. Schedules of Controlled Substances placement of brivaracetam into Schedule V. Interim final rule with request for comments. Fed. Regist. 81(92), 29487-29492 (2016).

67. Russo E, Citaro R, Mula M. The preclinical discovery and development of brivaracetam for the treatment of focal epilepsy. Expert Opin. Drug Discov. 12(11), 1169-1178 (2017)

68. American Academy of Neurology (AAN). Annual Meeting Abstracts Publication. www.aan.com

69. American Epilepsy Society (AES). AES meeting abstracts. 67th Annual Meeting of the American Epilepsy Society (AES). Washington, DC, USA, 6-10 December 2013. www.aesnet.org

70. Steinig I, von Podewils F, Moddel G et al. Post marketing experience with brivaracetam in the treatment of epilepsies: a multicenter cohort study from Germany. Epilepsia 58(7), 1208-1221 (2017).

-. A rigorous postmarketing study, see text for discussion.

71. Milovanocic JR, Jankovic SM, Pejcic A et al. Evaluation of brivaracetam: a new drug to treat epilepsy. Expert Opin. Pharmacother. 18, 1381-1389 (2017).

72. Klitgaard H, Matagne A, Nicholas JM et al. Brivaracetam: rationale for discovery and preclinical profile of a selective SV2A ligand for epilepsy treatment. Epilepsia 57, 538-548 (2016).

73. Finnema SJ, Mercier J, Naganawa M et al. Brivaracetam enters the brain faster than levetiracetam: a PET study in healthy volunteers. Neurology 88, p6.233 (2017).

74. Nicholas JM, Hannestad J, Holden D et al. Brivaracetam, a selective high-affinity synaptic vesicle protein 2A (SV2A) ligand with preclinical evidence of high brain permeability and fast onset of action. Epilepsia 57(2), 201-209 (2016).

75. Moseley BD, Sperling MR, Asadi-Pooya AA et al. Efficacy, safety, and tolerability of adjunctive brivaracetam for secondarily generalized tonic-clonic seizures: pooled results from three Phase III studies. Epilepsy Res. 127, 179-185 (2006).

76. Klein P, Tyrikova I, Brazdil M et al. Brivaracetam for the treatment of epilepsy. Expert Opin. Pharmacother. 17, 283-295 (2016).

77. Toledo M, Whitesides J, Schiemann J et al. Safety, tolerability, and seizure control during long-term treatment with adjunctive brivaracetam for partial-onset seizures. Epilepsia 57, 1139-1151 (2016).

78. Asadi-Pooya AA, Sperling MR, Chung $S$ et al. Efficacy and tolerability of adjunctive brivaracetam in patients with prior antiepileptic drug exposure: a post hoc study. Epilepsy Res. 131, 70-75 (2017).

79. Strzelczyk A, Steinig I, Willems L et al. Treatment of refractory and super-refractory status epilepticus with brivaracetam: a cohort study from two German university hospitals. Epilepsy Behav. 70(Pt A), 177-181 (2017). 
80. Niquet J, Suchomelova L, Thompson $\mathrm{K}$ et al. Acute and long-term effects of brivaracetam and brivaracetam-diazepam combinations in an experimental model of status epilepticus. Epilepsia 58(7), 1199-1207 (2017).

81. Brigo F, Bragazzi N, Nardone R et al. Efficacy and tolerability of brivaracetam compared to lacosamide, eslicarbazepine acetate, and perampanel as adjunctive treatments in uncontrolled focal epilepsy: results of an indirect comparison meta-analysis of RCTs. Seizure 42, 29-37 (2016).

82. Zhu L, Chen D, Xu D et al. Newer antiepileptic drugs compared to levetiracetam as adjunctive treatments for uncontrolled focal epilepsy: an indirect comparison. Seizure 51, 121-132 (2017).

83. French J. Will brivaracetam help my patient? Only time will tell. Epilepsy Curr. 17, 35-36 (2017).

84. Klein P. Author response to epilepsy current commentary "Will brivaracetam help my patient? Only time will tell." Neurology (2017). http://n.neurology.org/content/author-response-epilepsy-current-commentary-will-brivaracetam-help-my-patient-only-time-will

85. Kälviäiinen R, Genton P, Andermann E et al. Brivaracetam in patients with Unverricht-Lundborg disease: results from two randomized, placebo-controlled, double-blind studies. Epilepsia 50(Suppl. 10), 47 (2009).

86. 28th International Epilepsy Congress (IEC), Budapest, Hungary, 28 June-2 July 2009. 